\title{
Transport of drugs by the multidrug transporter AcrB involves an access and a deep binding pocket that are separated by a switch-loop
}

Eicher, Thomas ; Cha, Hi-jea ; Seeger, Markus A ; Brandstätter, Lorenz ; El-Delik, Jasmin ; Bohnert, Jürgen A ; Kern, Winfried V ; Verrey, François ; Grütter, Markus G ; Diederichs, Kay ; Pos, Klaas M

\begin{abstract}
AcrAB-TolC is the major efflux protein complex in Escherichia coli extruding a vast variety of antimicrobial agents from the cell. The inner membrane component AcrB is a homotrimer, and it has been postulated that the monomers cycle consecutively through three conformational stages designated loose $(\mathrm{L})$, tight $(\mathrm{T})$, and open $(\mathrm{O})$ in a concerted fashion. Binding of drugs has been shown at a periplasmic deep binding pocket in the $\mathrm{T}$ conformation. The initial drug-binding step and transport toward this drug-binding site has been elusive thus far. Here we report high resolution structures $(1.9-2.25 \AA)$ of AcrB/designed ankyrin repeat protein (DARPin) complexes with bound minocycline or doxorubicin. In the AcrB/doxorubicin cocrystal structure, binding of three doxorubicin molecules is apparent, with one doxorubicin molecule bound in the deep binding pocket of the $\mathrm{T}$ monomer and two doxorubicin molecules in a stacked sandwich arrangement in an access pocket at the lateral periplasmic cleft of the $\mathrm{L}$ monomer. This access pocket is separated from the deep binding pocket apparent in the T monomer by a switch-loop. The localization and conformational flexibility of this loop seems to be important for large substrates, because a G616N AcrB variant deficient in macrolide transport exhibits an altered conformation within this loop region. Transport seems to be a stepwise process of initial drug uptake in the access pocket of the $\mathrm{L}$ monomer and subsequent accommodation of the drug in the deep binding pocket during the $\mathrm{L}$ to $\mathrm{T}$ transition to the internal deep binding pocket of the $\mathrm{T}$ monomer.
\end{abstract}

DOI: https://doi.org/10.1073/pnas.1114944109

Posted at the Zurich Open Repository and Archive, University of Zurich

ZORA URL: https://doi.org/10.5167/uzh-62599

Journal Article

Accepted Version

Originally published at:

Eicher, Thomas; Cha, Hi-jea; Seeger, Markus A; Brandstätter, Lorenz; El-Delik, Jasmin; Bohnert, Jürgen A; Kern, Winfried V; Verrey, François; Grütter, Markus G; Diederichs, Kay; Pos, Klaas M (2012). Transport of drugs by the multidrug transporter AcrB involves an access and a deep binding pocket that are separated by a switch-loop. Proceedings of the National Academy of Sciences of the United States of America (PNAS), 109(15):5687-5692.

DOI: https://doi.org/10.1073/pnas.1114944109 

that are separated by a switch-loop

Thomas Eicher ${ }^{1,2 \#}$, Hi-jea Cha ${ }^{1 \#}$, Markus A. Seeger ${ }^{2,3 \#}$, Lorenz Brandstätter ${ }^{1,2}$, Jasmin El-Delik ${ }^{1}$, Jürgen

${ }^{1}$ Institute of Biochemistry and Cluster of Excellence Frankfurt - Macromolecular Complexes, GoetheUniversity Frankfurt, Max-von-Laue-Str. 9, D-60438 Frankfurt am Main, Germany.

${ }^{2}$ Institute of Physiology and Zurich Centre for Integrative Human Physiology (ZIHP), University of Zurich, Winterthurerstrasse 190, CH-8057 Zürich, Switzerland.

${ }^{3}$ Institute of Biochemistry, University of Zurich, Winterthurerstrasse 190, CH-8057 Zürich, Switzerland.

${ }^{4}$ Center for Infectious Diseases and Travel Medicine, University Hospital, Department of Medicine, Albert-Ludwigs University, Freiburg, Germany

${ }^{5}$ Department of Biology, University of Konstanz, Universitätsstrasse 10, M647, D-78457 Konstanz, Germany

*To whom correspondence should be sent:

K. M. Pos: tel. +49-69-798-29251, Fax: +49-69-798-29201

E-Mail: pos@em.uni-frankfurt.de

\# These authors contributed equally

Acknowledgments:

This work was supported by the Swiss National Foundation, the German Research Foundation (SFB 807, Transport and Communication across Biological Membranes) to K. M. P. and the Forschungskredit der Universität Zürich to L.B. Support by the DFG-EXC115 (Cluster of Excellence Macromolecular Complexes at the Goethe-University Frankfurt) is gratefully acknowledged by K.M.P. We thank the beamline staff at the Swiss Light Source (PXI, SLS) of the Paul Scherrer Institut in Villigen (Switzerland). The atomic coordinates and structure factors of the DARPin complexes of wt AcrB (in complex with minocycline), AcrB_G616N and wt AcrB (in complex with doxorubicin) have been deposited at the Protein Data Bank with accession numbers 4DX5, 4DX6, and 4DX7, respectively.

Biological Sciences: Biochemistry 
1 AcrAB-TolC is the major efflux protein complex in Escherichia coli extruding a vast variety of 2 antimicrobial agents from the cell. The inner membrane component AcrB is a homotrimer and it has 3 been postulated that the monomers cycle consecutively through three conformational stages 4 designated loose (L), tight (T) and open (O) in a concerted fashion. Binding of drugs has been shown 5 at a periplasmic deep binding pocket in the T conformation. The initial drug binding step and 6 transport towards this drug binding site has been elusive thus far.

7 Here we report high resolution structures (1.9-2.25 Å) of AcrB/DARPin complexes with bound 8 minocycline or doxorubicin. In the AcrB/doxorubicin co-crystal structure, binding of three doxorubicin molecules is apparent, with one doxorubicin molecule bound in the deep binding pocket of the T monomer and two doxorubicin molecules in a stacked sandwich arrangement in an access pocket at the lateral periplasmic cleft of the $L$ monomer. This access pocket is separated from the deep binding pocket apparent in the T monomer by a switch-loop. The localization and conformational flexibility of this loop appears to be important for large substrates, as a G616N AcrB variant deficient in macrolide transport exhibits an altered conformation within this loop region. Transport appears to be a stepwise process of initial drug uptake in the access pocket of the $L$ monomer and subsequent accommodation of the drug in the deep binding pocket during the $L$ to $T$ transition to the internal deep binding pocket of the T monomer. 
2 The three component AcrA/AcrB/TolC multidrug resistance (MDR) pump provides the Gram-negative

3 E. coli cell with the necessary means to protect itself against a wide range of noxious compounds (1).

4 AcrB resides in the inner membrane and is the energy transducing and substrate specificity determinant of the entire three component pump assembly $(2,3)$. AcrA is the adapter component which associates the inner membrane pump with the TolC outer membrane channel $(4,5)$. Importantly, all three components are necessary to obtain the MDR phenotype $(3,4)$. The first de novo AcrB crystal structure was solved via X-ray crystallography at $3.5 \AA$ resolution by Murakami et al. (6) in 2002 (PDB entry: 1IWG) and showed an ligand-free homotrimeric assembly (Figure S1AB). Each monomer contains a twelve transmembrane helices (TM)-containing transmembrane domain with TM4 and TM10 harbouring essential titratable residues (D407, D408 and K940) inside the topological core (7)(Figure S1C). Approximately half of the protein's 1049 amino acids forms two extensive periplasmic loops connecting TM1 with TM2 and TM7 with TM8 and extends $70 \AA$ into the periplasm, comprising the TolC docking domain and a porter domain (Figure S1AB). The latter domain is divided in subdomains PN1, PN2, PC1 and PC2, which are coupled by sequential proximity (PN1-PN2, PC1-PC2) and by sharing $\beta$-strands to form common $\beta$-sheets (PN2-PC1, PC2-PN1)(Figure S1D). Between the PC1 and PC2 subdomains, a cleft is apparent oriented approximately perpendicular to the membrane plane (Figure S1BD). In the centre of the trimer, the TolC docking domain exhibits a funnel-like structure narrowing to a central pore, defined by $\alpha$-helices (designated pore helices) of the PN1 subdomains of each monomer. This pore has a small diameter and does not allow drugs to pass. Towards the membrane plane, the central pore leads to a central cavity and further to a 30 to $35 \AA$ wide, presumably lipid-filled transmembrane hole defined by the ring-like arrangement of the TM helices of the trimer (Figure S1). Three vestibules at the monomer interface located just above the membrane plane lead towards the central cavity (Figure S1AB). The periplasmic boundaries of these vestibule areas were recently shown to be accessible for the AcrB substrate Bodipy FL $N$-(2-aminoethyl) maleimide (8). Moreover, the substrate specificity of the entire AcrAB-TolC efflux machinery was found to be localized in the AcrB periplasmic porter domain (2), whereas the AcrB transmembrane domain appears to be essential for proton translocation (7, 9)(Figure S1). In 2006, three groups independently published an asymmetric structure of AcrB grown in the monoclinic space group C2 (10-12) (PDB entries: 2DHH, 2DR6, 2DRD, 2GIF, 2.8 to $2.9 \AA$ A), triclinic space group P1 (11) (2HRT, $3.0 \AA$ ) and an AcrB structure including bound designed ankyrin repeat proteins 33 (DARPins) grown in orthorhombic space group $\mathrm{P} 2{ }_{1}{ }_{2}{ }_{1} 2_{1}(2 \mathrm{~J} 8 \mathrm{~S}, 2.5 \AA)(12)$. Within the asymmetric AcrB 34 trimer, each monomer has a different conformation denoted as loose (L), tight ( $T$ ) and open (O) 35 (access, binding and extrusion, respectively) (10-12), with the L (or access) state closest to the 
1 conformation of the monomers in the symmetric structure (6). Two of the deposited asymmetric

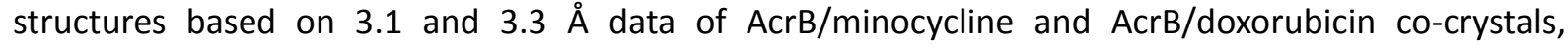
respectively (10), showed binding of (9-bromo) minocycline or doxorubicin to a hydrophobic binding pocket exclusively present in one of the monomers (the $T$ or binding monomer) within the asymmetric AcrB trimer. The DARPin bound AcrB structure was almost identical to the AcrB structures crystallized without binder with root mean square deviation (rmsd) of the superimposed trimeric structures $\leq 1 \AA$. The DARPin molecules only bind to the $L$ and $T$ conformers, resulting in a ratio of two DARPin molecules per AcrB trimer. This stoichiometry was verified by densitometry after microchannel electrophoresis, sedimentation velocity experiments and via laser-induced liquid bead ion desorption mass spectrometry (LILBID-MS), suggesting that in solution and probably also in vivo AcrB adopts mainly the asymmetric conformation $(12,13)$. In analogy with the functional rotation of the $\alpha / \beta$-subunits of the $F_{1} F_{0}$ ATPase leading to synthesis of ATP (14), a similar catalytic mechanism was postulated for AcrB leading to drug export (10-12). In short, the hypothesis states that within the asymmetric trimer, the monomers can adopt any of the conformational states $\mathrm{L}, \mathrm{T}$ and $\mathrm{O}$. Transport depends on the concerted - but not necessarily synchronous - cycling of the monomers through the states $\mathrm{L}, \mathrm{T}, \mathrm{O}$ and back to $\mathrm{L}$, which is the essence of the functional rotation. In the $L$ conformation, substrates are recruited from the periplasmic space and/or the membrane for transport during the $L$ to $T$ transition towards the deep substrate binding pocket inside the porter domain. Upon conformational change towards the $\mathrm{O}$ state, substrate is released from the binding pocket and exits AcrB via its central funnel towards the TolC tunnel. During a complete functional rotation, occlusions and constrictions inside the porter domain seem to propagate from a lateral opening towards the central funnel driving the unidirectional transport of substrate. Hence, this mechanism was dubbed "peristaltic pump mechanism" $(11,15,16)$ (Figure S1EFG). The conversion from $T$ to $O$ is suggested to be the energy-requiring step, in analogy to the binding change mechanism for the $F_{1} F_{0}$ ATPase (14). Drug efflux is also subject of bi-site activation i.e. the $\mathrm{T}$ to $\mathrm{O}$ transition within the LTO trimer (and the resulting release of the drug) only occurs when a second substrate has bound to the adjacent monomer. Strengthening this view are the recently observed strong cooperative kinetics of the extrusion of $\beta$-lactam antibiotics by the AcrAB-TolC system $(17,18)$.

The functional rotation mechanism suggests interdependence of the monomers, which means that inactivation of only one of the monomers will render the entire trimer inactive. Recently, this has been impressively shown to be the case by Nikaido and co-workers by the use of a covalently linked AcrB trimer (19). The proposed conformational cycling and the substantial rearrangement of the 
1 formation of disulfide bonds significantly decreased AcrB mediated transport and restoring activity by

2 the addition of the reducing reagent dithiothreitol (DTT).

3 Two conformationally distinct trimeric AcrB structures are known until now: the symmetric LLL (all 4 loose) conformation (6) and the asymmetric LTO conformation (10-12). The very recently published crystal structure of the $\mathrm{Cu}^{+}$and $\mathrm{Ag}^{+}$transporter CusA from E. coli showed substrates bound to the periplasmic porter domain in all three protomers adapting a symmetric conformation with structural features describing a TTT conformation (22). Most of the published AcrB structures are in the symmetric conformation, but show in particular cases slight deviations between each other, indicating intrinsic flexibility $(6,7,23-26)$. The LLL conformation has been postulated the "resting state" -in the absence of substrate- $(7,15)$ with the structural flexibility necessary for substrate acquisition (25). Recently published symmetric LLL state structures (at 3.85-3.2 Å resolution) were shown to accommodate substrates to the inner wall of the transmembrane cavity (25-27) or in the open cleft determined by the PC1/PC2 subdomains, constituting the access to tunnel 2 (Figure S1EF) $(24,27)$. One transport hypothesis assumes the substrate to be garnered from the outer leaflet of the inner membrane or directly from the periplasm. Substrates which are partitioned in the outer leaflet of the inner membrane might enter the $\mathrm{L}$ monomer via TM8/TM9 groove whereas substrates located in the periplasm might enter the $\mathrm{L}$ monomer via the PC1/PC2 cleft i.e. the entrance of tunnel 2 approximately $15 \AA$ above the membrane plane (Figure S1E). Also a concerted and consecutive drug uptake via the TM8/TM9 groove and subsequent transport to the PC1/PC2 cleft might be envisioned. High resolution ( $2.5 \AA$ ) data describing the asymmetric trimer show clear densities in the TM8/TM9 groove of the $L$ monomer, which has been attributed to the highly concentrated detergent and AcrB substrate $n$-dodecyl- $\beta$-D-maltoside (12). Upon conformational change from the $L$ to the $T$ state, tunnel 1 appears and might provide one of the pathways for substrates towards the hydrophobic deep binding pocket which accommodates the substrate molecule. Upon transition from the $T$ to the $O$ state, the binding pocket closes again; substrate is squeezed out and follows a newly formed tunnel (tunnel 3 ) to the funnel, and is from there finally extruded into the media via TolC (Figure S1).

In the work presented here, high resolution structures (1.9 and $2.26 \AA$ ) describe a potential initial binding step of doxorubicin in the $\mathrm{L}$ conformer, and multiple substrate (minocycline or doxorubicin and dodecyl- $\alpha$-D-maltoside) binding to the T conformer. Access to the internal deep binding pocket in the $T$ monomer is mediated by the conformational change from the $L$ to the $T$ conformation. In the $L$ monomer, two doxorubicin molecules are positioned in an access pocket and separated from the internal binding pocket by a flexible loop, designated switch-loop, containing F615 and F617. The 
1 pocket in the $\mathrm{L}$ conformation and subsequent transport to the deep binding pocket by the 2 conformational change of the switch-loop and the PN2/PC1 subdomains during the $L$ to $T$ transition. 


\section{Binding of minocycline and doxorubicin by the AcrB wild-type protein}

We describe here the crystallization and structural elucidation of AcrB/DARPin complexes in the presence of minocycline and doxorubicin based on $1.9 \AA$ and $2.25 \AA$ diffraction data (Table S1). AcrB/DARPin complexes were co-crystallized in the presence of minocycline $(2.0 \mathrm{mM})$ or doxorubicin $(0.4 \mathrm{mM})$, yielding orthorhombic crystals. The structures were refined to an $\mathrm{R}_{\text {free }}$ of $23.1 \%$ and $22.7 \%$, respectively, and describe an asymmetric trimer comprising three different monomer conformations representing the consecutive states loose $(\mathrm{L})$, tight $(\mathrm{T})$ and open $(\mathrm{O})(15)$. Electron densities for minocycline or doxorubicin could be clearly identified in the hydrophobic deep binding pocket of the T monomer (Figure 1, Figure S2). Whereas the position of minocycline is congruent with the structure of Murakami et al. (10), the orientation of the doxorubicin molecule inside the deep binding pocket differs substantially (Figure S2, Table S2). Notably, differences in crystallization conditions and resolution, and our use of crystallization chaperones (DARPins) are possible reasons for the observed distinctions. In the T monomer, another density located in the lateral cleft determined by the PC1 and PC2 subdomains of the periplasmic porter domain could be assigned to dodecyl- $\alpha$-D-maltoside (Figure S3). In the L monomer of the AcrB/doxorubicin co-crystal structure, but not in the AcrB/minocycline co-crystal structure, large densities were observed at the PC1/PC2 subdomain cleft (access pocket) and could be assigned to a two-fold symmetric sandwich of stacked doxorubicin molecules (Figure 2, Figure S4). The AcrB/doxorubicin co-crystal structure therefore reveals an asymmetric AcrB trimer containing three doxorubicin molecules, two doxorubicin molecules bound to the access pocket in the $L$ monomer and one bound to the deep binding pocket in the T monomer (Figure S5). This novel observation indicates a possible link between the initial binding of drugs in the $L$ monomer and the subsequent binding of the drug in the deep binding pocket mediated by the $L$ to $\mathrm{T}$ transition in the functional rotation cycle (Figure S1EFG, Figure S5).

Coordination of doxorubicin by residues inside the AcrB access pocket includes hydrophobic interactions with F666 and L828 and charged/polar interactions with T676, R717 and N719 (Figure S3). In a recent study, these coordination sites and further proximate residues (D566, F664, L668 and E673, Figure S4) were Cys-substituted and accessible to the AcrB substrate Bodipy FL maleimide (28), matching the crystallographic data presented here (Figure S4B). Furthermore, single alanine substitution of residues F664, F666, E673 and R717 markedly interfered with the ability of these AcrB variants to confer resistance towards various toxic compounds (27).

34 The $L$ monomer is considered the access protomer $(10,11)$ involved in the initial stage of drug 35 binding. The lateral access pocket at the periplasmic cleft confined by the PC1 and PC2 subdomains 
1 might be the initial entry point for drugs to the AcrB functional rotation cycle. However, many of the AcrB substrates are expected also to partition into the outer leaflet of the inner membrane due to their hydrophobic properties. A suggested entry pathway for drugs partitioned in the outer leaflet of the inner membrane is the hydrophobic groove in the TM domain defined by TM8 and TM9 $(6,15)$, where in the high resolution structures presented here, binding of the detergent and AcrB substrate dodecyl- $\beta$-D-maltoside is apparent (Figure S6).

The TM8/TM9 groove defines the entrance to tunnel 1 (Figure S1F) $(12,15)$ inside the periplasmic porter domain, which leads towards the hydrophobic deep binding pocket (10-12). This tunnel and the opening of the deep binding pocket with subsequent minocycline or doxorubicin binding are apparent after the $\mathrm{L}$ to $\mathrm{T}$ transition (Figure 1 and (10)). The $\mathrm{L}$ monomer of the AcrB/doxorubicin structure comprises one dodecyl- $\beta$-D-maltoside molecule at the TM8/TM9 transmembrane groove and a doxorubicin dimer sandwich at the access pocket (Figure S4, FigureS6). The access pocket and the TM8/TM9 groove might represent exclusive entry points for drugs with different properties, or consecutive gates in a multistep transport mechanism.

\section{A switch-loop separates access and deep binding pocket areas}

The doxorubicin molecules in the access pocket of the $L$ monomer are separated from the deep binding pocket (Figure 1) by a loop containing F615, F617 and R620 (switch-loop, Figure 2AB).

The switch-loop adopts alternative conformations in the $\mathrm{L}$ and $\mathrm{T}$ monomer. It appears to modulate the path from access pocket in the L conformation to the deep binding pocket in the T conformation. Superimposition of the $\mathrm{L}$ and $\mathrm{T}$ conformations indicates that the side chain conformation of F615 (located on the switch-loop) and Q176 (located on the PN2 subdomain) seen in the L monomer would interfere with doxorubicin binding in the deep binding pocket and the switch-loop main chain conformation in the T conformation would sterically interfere with the stacked doxorubicin location as observed in the $L$ conformation. Therefore, steric hindrance presumably precludes binding of the drugs in the deep binding pocket in the $L$ monomer, whereas the alternative loop conformation appears to prevent binding of doxorubicin to the access pocket in the T conformation (Figure 2B). The switch-loop conformation in the structure of the symmetric AcrB (i.e. the LLL conformation) is less inward oriented and would theoretically interfere with doxorubicin binding if these would localize at the same position as observed in the asymmetric LTO AcrB trimer (Figure S7). Substrates like ethidium (pdb entry: 1T9X, $3.1 \AA$ A)(29) or taurocholate (pdb entry: 2W1B, $3.85 \AA$ ) $(24)$ appear to bind more at the periphery of the PC1/PC2 cleft compared to doxorubicin binding in the asymmetric cocrystal structure (Figure S7C).

It was recently reported (30) that a single substitution in the switch loop (G616N) decreases AcrB's ability to extrude macrolides, resembling the poor efflux activity towards macrolides shown by the 
1 Pseudomonas aeruginosa homologue MexB (wildtype MexB contains N616). The switch-loop 2 conformation in the L conformation of the AcrB G616N variant (solved at $2.9 \AA$ in the presence of 3 minocycline, Table S1) resembles the loop conformation of wildtype AcrB in the T monomer and of the switch-loop conformation found in the wildtype MexB L monomer structure (Figure 2BC). The differences in the switch-loop conformations between the wildtype AcrB and G616N variant are clearly defined by the differences in electron density in this region of the protein (Figure S6CD). Superimposition of the $L$ monomer structure of MexB or AcrB G616N variant on the AcrB wildtype $L$ monomer structure predicts a minor steric clash with one of the doxorubicin molecules bound the access pocket of wildtype AcrB (Figure 2C). We conducted a drug resistance assay on solid medium using E. coli BW25113 $\triangle \mathrm{acrB}$ comprising wildtype or G616N AcrB equally well-expressed from plasmids (Figure S8). Clearly, an effect of the G616N substitution on the resistance against erythromycin could be detected, and a subtle difference in growth in the presence of doxorubicin was observed. Growth on other substrates like novobiocin, ethidium or chloramphenicol was, however, also slightly affected by the substitution in various degrees. In MIC experiments reported recently (30) using liquid media and chromosomal substitution of the G616N variant in an E. coli AG100 background, larger macrolide molecules are substantially less well transported by this variant whereas other substrates like novobiocin, ethidium and chloramphenicol showed wildtype resistance. Specific sensitivity towards macrolides was also conferred when F615 (localized on the switch-loop) was substituted with Ala, or when residues 615-617 were deleted from the loop (31).

Access, Binding and Extrusion, the three cyclic actions mediated by the loose, tight and open monomer

The structural information obtained in this study from crystallization and structural elucidation of the wildtype AcrB with bound minocycline and doxorubicin at unprecedented high resolution, as well as the structures of the AcrB variant $G 616 \mathrm{~N}$ can be combined in a model for access, binding and extrusion of drugs catalyzed by AcrB. Homotrimeric AcrB can adopt three different monomer conformations representing the consecutive states $L, T$ and $O$. However, during transition of the conformations within the trimer, AcrB is anticipated to exist in intermediate states e.g. TTO $(15,16)$ a hypothesis that is supported by quantitative cysteine cross-link experiments and molecular dynamics studies $(21,32)$.

31 The LTO conformation most likely represents the lowest energy form of the AcrB trimer in the 32 presence of substrate $(15,16,32)$, since in vivo cross-link studies (21) and analytical 33 ultracentrifugation experiments in the presence of DARPins $(12,13)$ imply the presence of this 34 conformation in the membrane and in detergent rather than the readily crystallizable LLL form. The 35 latter state might preferably exist in the absence of ligands (i.e. "resting state") as has been suggested 
1 previously $(7,15,32)$ and apparently is preferred within the R32 crystal lattice. Once substrates are 2 present in the periplasmic space, and/or partitioned in the periplasmic space/inner-membrane 3 boundary, AcrB-catalyzed efflux of the drugs over the outer membrane is counteracting the influx. The $L$ monomer provides probable initial binding sites for drugs to enter the AcrB protein. One entry point might be the TM8/TM9 groove located at the height of the periplasm/membrane boundary i.e. the outer leaflet of the inner membrane. In the high resolution structures presented here (Figure S6) and in the $2.5 \AA$ structure by Sennhauser et al. (12), a dodecyl- $\beta$-D-maltoside molecule is deeply buried inside the TM8/TM9 transmembrane groove unlike the other more loosely bound dodecyl- $\beta$ D-maltoside molecules which were assigned from the data of the electron density maps. Moreover, this groove is located exactly below tunnel 2 (Figure S1F) in the T monomer leading to the deep binding pocket $(12,15)$. In addition, recent Bodipy FL $N$-(2-aminoethyl) maleimide labeling data indicated that residue S836, located at the entrance of tunnel 2 on the PC2 subdomain was highly accessible for this AcrB substrate (8). A second entry point is postulated at a lateral access pocket at the periplasmic cleft confined by the PC1 and PC2 subdomains (Figure S1BD, Figure 2, Figure S4, Figure S6). Both putative entry sites might represent exclusive access points for drugs with different properties, or consecutive binding sites. Interestingly, both sites collapse during the proton motive force dependent $\mathrm{T}$ to $\mathrm{O}$ transition simultaneous with the closure of the deep binding pocket and the disappearance of the tunnels leading from both these putative drug entry points towards the deep binding pocket $(11,12,15)$. The postulated entry step at the periplasmic cleft is depicted by the doxorubicin sandwich inside the access pocket of the $\mathrm{L}$ monomer (Figure 3A). Binding of substrates (ethidium, rhodamine 6G, ciprofloxacin, nafcillin, Phe-Arg- $\beta$-naphthylamide and taurocholate) was also shown in the symmetric L-state trimer $(24,29)$ at a more lateral position of the PC1/PC2 cleft (Figure S7). The more lateral binding might indicate an earlier step in substrate acquisition by the complete ligand-free "restingstate" LLL trimer or might indicate the promiscuity of binding different drugs to different sites within the cleft area. While it is unclear why two doxorubicin molecules are bound to the $L$ monomer of the asymmetric trimer, it appears that one of the drug molecules can be further transported deeper into the AcrB protein towards the T monomer deep binding pocket (Figure 3B). Very recently, co-crystal structures from 3.3 $\AA$ X-ray data showed the binding of high molecular mass drugs erythromycin and rifampicin to the access pocket, whereas the low molecular mass substrates like minocyclin and doxorubicin were postulated to be transported directly to the deep binding pocket, without binding first to the access pocket (33). Since doxorubicin is mainly present as dimer in solution at concentrations where the AcrAB-TolC efflux system confers resistance to $E$. coli against the drug (and at the concentrations used for co-crystallization)(34), the binding in a dimeric state in the access pocket might represent a preliminary stage to the binding of the doxorubicin monomer in the deep 
1 binding pocket. This entails prerequisite conformation flexibility between the $L$ and $T$ monomer, as 2 well as a binding affinity difference of the access pocket (low affinity) and deep binding pocket (high 3 affinity). The switch-loop and the $\beta$-sheets of the PN2/PC1 subdomains prohibit entry of the drug in the deep binding pocket in the $L$ conformation (Figure 2) and rearrangement of these structures in the $L$ to $T$ transition facilitate access to the deep binding pocket in the $T$ monomer (Figure 3B). The presence of dodecyl- $\alpha$-D-maltoside in the PC1/PC2 cleft of the T monomer appears to contradict the predicted function of the switch-loop. The maltoside moiety of dodecyl- $\alpha$-D-maltoside is localized at the same position as the daunosamine sugar ring of one of the doxorubicin molecules in the L monomer, whereas the aliphatic tail protrudes into the porter domain and appears not to interfere with the switch loop conformation in the T monomer (Figure S3). The presence of dodecyl- $\alpha$-Dmaltoside at this position in the T monomer is puzzling, even more so since the high-resolution of the electron density maps did allow to clearly distinguishing between the $\alpha$-and $\beta$-anomers of this detergent. Since only low concentrations of $\alpha$-anomer are present in the commercial batch of dodecyl- $\beta$-D-maltoside, the binding must occur with high affinity of this ligand. Once binding of drug to the deep binding pocket occurs, it is most likely stabilizing the T conformation and is enabling further stabilization by the PN1 subdomain tilting of the neighbouring monomer (Figure 3B). Thus, movements of the subdomains PN2/PC1 in the $L$ to $T$ transition not only takes effect on further conformational changes in the same monomer (i.e. $T$ to $O$ transition)(Figure 3C), but is also implied to have an effect on the conformational changes in the neighbouring monomers. Movement of the subdomains during the $T$ to $O$ transition is coupled to an extensive kinking of TM8, which appears to be coupled to conformational changes in the transmembrane domain. Most likely protonation events i.e. uptake of protons from the periplasm to the proton relay residues (D407/D408) located in the transmembrane domain lead to a conformational change of the TM helices and this movement is presumably transduced to TM8, which is connected to the PN1/PC2 subdomains. Once the drug has been released from the $O$ conformation, a switch to the $L$ conformation to initiate another round of drug binding in the access pocket appears necessary (Figure 3D). The reorientation of the PN1/PC2 subdomains during the $O$ to $L$ transition leads to dekinking of TM8, which possibly triggers the deprotonation events in the TM domain, stabilizing the $\mathrm{L}$ monomer conformation and a new round of antibiotic uptake via the TM8/TM9 groove and/or access pocket can be started (Figure 3). Molecular insight into coupling of the protonation and deprotonation events in the transmembrane domain to the conformational changes in the periplasmic porter domain leading to drug efflux will eventually provide the complete chain of events of proton motive force-driven drug extrusion by AcrB. 


\section{Bacterial strains, plasmids and growth conditions}

E. coli $\mathrm{DH} 5 \alpha$ was used as host for cloning procedures. E. coli C43 (DE3)(35) harboring pET24acrB $\mathrm{His}_{\mathrm{H}}$

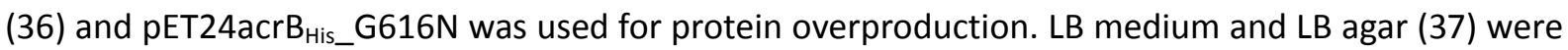
used for routine bacterial growth at $37^{\circ} \mathrm{C}$. Kanamycin (Applichem) was used at $50 \mu \mathrm{g} \mathrm{m}^{-1}\left(\mathrm{Kan}^{50}\right)$. Drug resistance was assayed using solid media, as described previously (38).

\section{Site directed mutagenesis}

$\mathrm{pET} 24 \mathrm{acrB}_{\mathrm{His}}$ (36) served as a template for site-directed mutagenesis. By using the Quikchange protocol (Stratagene), amino acid substitution was achieved with $5^{\prime}$ phosphorylated primers. Insertion of mutation was verified by sequencing (Microsynth, Balgach, Switzerland).

\section{Crystallization of AcrB wildtype and G616N in presence of DARPins}

Overexpression and purification of DARPin clone 1108_19 was accomplished as described (12). AcrB wildtype and AcrB_G616N were overproduced based on the method reported previously $(11,36)$ with the following variations for G616N. Expression was induced with IPTG ( $1 \mathrm{mM}$ )at $\mathrm{OD}_{600}=0.8$ and the culture was incubated overnight at $20^{\circ} \mathrm{C}$ producing up to $20 \mathrm{mg}$ AcrB_G616N per L culture after affinity chromatography. Due to the tendency of AcrB_G616N to aggregate, the protein concentration for crystallization was experimentally determined to be optimal at $8 \mathrm{mg} \mathrm{ml}^{-1}$. DARPins synthesis, purification and AcrB/DARPin complex crystallization was done according to Sennhauser et al. (12) using n-dodecyl- $\beta$-D-maltoside (Glycon, Germany, with $0.01 \% \alpha$-isomer). Substrate was added prior to crystallization at concentrations of $2 \mathrm{mM}$ and $0.4 \mathrm{mM}$ for minocycline or doxorubicin, respectively. Crystals (AcrB/DARPin and G616N/DARPin complex) were grown by the hanging drop vapor diffusion method at $0.05 \mathrm{M} \mathrm{N}$-(2-Acetamido)-iminodiacetic acid (ADA) buffer, pH 6.5, 7-9\% polyethylene glycol (PEG) 4000, 6-10\% glycerol and $0.2 \mathrm{M}\left(\mathrm{NH}_{4}\right)_{2} \mathrm{SO}_{4}$. AcrB_G616N/DARPin crystals were grown in the presence of minocycline ( $2 \mathrm{mM}$ final concentration), but electron densities at 2.9 $\AA$ at the expected deep binding pocket location were too weak to assign minocycline binding at this position. 
2 Datasets of $\mathrm{P} 2{ }_{1} 2_{1} 2_{1}$ crystals were collected at the beamline X06SA of the Swiss Light Source (Paul

3 Scherrer Institut, Villigen, Switzerland) (wavelength 0.8-1.0 A). Data reduction was done with the XDS 4 package (39). The structures were solved by molecular replacement using MOLREP (40) or PHASER

5 (41). Refinement was performed with the program phenix.refine from the PHENIX package (42) using 6 using rigid body refinement followed by restrained refinement with TLS restraints. Model rebuilding was performed using the program СОOT (43). For superimposition of the AcrB monomers and ligands, the program SUPERPOSE (44) was used matching the DN (residues 181-272) and DC (residues 724812) subdomains (11). Figures were created using Pymol (http://pymol.sourceforge.net/).

\section{References}

1. Nikaido H \& Takatsuka Y (2009) Mechanisms of RND multidrug efflux pumps. Biochim Biophys Acta 1794(5):769-781.

2. Elkins CA \& Nikaido H (2002) Substrate specificity of the RND-type multidrug efflux pumps AcrB and AcrD of Escherichia coli is determined predominantly by two large periplasmic loops. J Bacteriol 184(23):6490-6498.

3. Ma D, et al. (1995) Genes acrA and acrB encode a stress-induced efflux system of Escherichia coli. Mol Microbiol 16(1):45-55.

4. Fralick JA (1996) Evidence that TolC is required for functioning of the Mar/AcrAB efflux pump of Escherichia coli. J Bacteriol 178(19):5803-5805.

5. Symmons MF, Bokma E, Koronakis E, Hughes C, \& Koronakis V (2009) The assembled structure of a complete tripartite bacterial multidrug efflux pump. Proc Natl Acad Sci U S A 106(17):7173-7178.

6. Murakami S, Nakashima R, Yamashita E, \& Yamaguchi A (2002) Crystal structure of bacterial multidrug efflux transporter AcrB. Nature 419(6907):587-593.

7. Su CC, et al. (2006) Conformation of the AcrB multidrug efflux pump in mutants of the putative proton relay pathway. J Bacteriol 188(20):7290-7296.

8. Husain F, Bikhchandani M, \& Nikaido H (2011) Vestibules are part of the substrate path in the multidrug efflux transporter AcrB of Escherichia coli. J Bacteriol 193(20):5847-5849.

9. Seeger MA, von Ballmoos C, Verrey F, \& Pos KM (2009) Crucial role of Asp408 in the proton translocation pathway of multidrug transporter AcrB: evidence from site-directed mutagenesis and carbodiimide labeling. Biochemistry 48(25):58015812.

10. Murakami S, Nakashima R, Yamashita E, Matsumoto T, \& Yamaguchi A (2006) Crystal structures of a multidrug transporter reveal a functionally rotating mechanism. Nature 443(7108):173-179.

11. Seeger MA, et al. (2006) Structural asymmetry of AcrB trimer suggests a peristaltic pump mechanism. Science 313(5791):1295-1298. 
12. Sennhauser G, Amstutz P, Briand C, Storchenegger O, \& Grutter MG (2007) Drug export pathway of multidrug exporter AcrB revealed by DARPin inhibitors. PLoS Biol 5(1):e7.

13. Brandstatter L, et al. (2011) Analysis of AcrB and AcrB/DARPin ligand complexes by LILBID MS. Biochim Biophys Acta 1808(9):2189-2196.

14. Boyer PD (1997) The ATP synthase $\mathrm{e}^{--}$a splendid molecular machine. Annu. Rev. Biochem. 66:717-749.

15. Pos KM (2009) Drug transport mechanism of the AcrB efflux pump. Biochim Biophys Acta 1794(5):782-793.

16. Seeger MA, et al. (2008) The AcrB efflux pump: conformational cycling and peristalsis lead to multidrug resistance. Curr Drug Targets 9(9):729-749.

17. Lim SP \& Nikaido H (2010) Kinetic parameters of efflux of penicillins by the multidrug efflux transporter AcrAB-TolC of Escherichia coli. Antimicrob Agents Chemother 54(5):1800-1806.

18. Nagano K \& Nikaido H (2009) Kinetic behavior of the major multidrug efflux pump AcrB of Escherichia coli. Proc Natl Acad Sci U S A 106(14):5854-5858.

19. Takatsuka Y \& Nikaido H (2009) Covalently linked trimer of the AcrB multidrug efflux pump provides support for the functional rotating mechanism. J Bacteriol 191(6):1729-1737.

20. Takatsuka Y \& Nikaido H (2007) Site-directed disulfide cross-linking shows that cleft flexibility in the periplasmic domain is needed for the multidrug efflux pump AcrB of Escherichia coli. J Bacteriol 189(23):8677-8684.

21. Seeger MA, et al. (2008) Engineered disulfide bonds support the functional rotation mechanism of multidrug efflux pump AcrB. Nat Struct Mol Biol 15(2):199-205.

22. Long F, et al. (2010) Crystal structures of the CusA efflux pump suggest methionine-mediated metal transport. Nature 467(7314):484-488.

23. Das D, et al. (2007) Crystal structure of the multidrug efflux transporter AcrB at 3.1A resolution reveals the N-terminal region with conserved amino acids. J Struct Biol 158(3):494-502.

24. Drew D, et al. (2008) The structure of the efflux pump AcrB in complex with bile acid. Mol Membr Biol 25(8):677-682.

25. Tornroth-Horsefield S, et al. (2007) Crystal structure of AcrB in complex with a single transmembrane subunit reveals another twist. Structure 15(12):1663-1673.

26. Yu EW, McDermott G, Zgurskaya HI, Nikaido H, \& Koshland DE, Jr. (2003) Structural basis of multiple drug-binding capacity of the AcrB multidrug efflux pump. Science 300(5621):976-980.

27. Yu EW, Aires JR, McDermott G, \& Nikaido H (2005) A periplasmic drug-binding site of the AcrB multidrug efflux pump: a crystallographic and site-directed mutagenesis study. J Bacteriol 187(19):6804-6815.

28. Husain F \& Nikaido H (2010) Substrate path in the AcrB multidrug efflux pump of Escherichia coli. Mol Microbiol 78(2):320-330.

29. Yu EW, Aires JR, McDermott G, \& Nikaido H (2005) A periplasmic drug-binding site of the AcrB multidrug efflux pump: a crystallographic and site-directed mutagenesis study. J. Bacteriol. 187(19):6804-6815. 
30. Wehmeier C, Schuster S, Fahnrich E, Kern WV, \& Bohnert JA (2009) Site-directed mutagenesis reveals amino acid residues in the Escherichia coli RND efflux pump AcrB that confer macrolide resistance. Antimicrob Agents Chemother 53(1):329330.

31. Bohnert JA, et al. (2008) Site-directed mutagenesis reveals putative substrate binding residues in the Escherichia coli RND efflux pump AcrB. $J$ Bacteriol 190(24):8225-8229.

32. Yao XQ, Kenzaki H, Murakami S, \& Takada S (2010) Drug export and allosteric coupling in a multidrug transporter revealed by molecular simulations. Nat Commun $1(8): 117$.

33. Nakashima R, Sakurai K, Yamasaki S, Nishino K, \& Yamaguchi A (Structures of the multidrug exporter AcrB reveal a proximal multisite drug-binding pocket. Nature 480(7378):565-569.

34. Menozzi M, Valentini L, Vannini E, \& Arcamone F (1984) Self-association of doxorubicin and related compounds in aqueous solution. J Pharm Sci 73(6):766-770.

35. Miroux B \& Walker JE (1996) Over-production of proteins in Escherichia coli: mutant hosts that allow synthesis of some membrane proteins and globular proteins at high levels. J Mol Biol 260(3):289-298.

36. Pos KM \& Diederichs K (2002) Purification, crystallization and preliminary diffraction studies of AcrB, an inner-membrane multi-drug efflux protein. Acta Crystallogr. Biol. Crystallogr. D58(Pt 10 Pt 2):1865-1867.

37. Sambrook J, Fritsch EF, \& Maniatis T (1989) Molecular cloning: a laboratory manual (Cold Spring Harbor Laboratory Press, Cold Spring Harbor, N.Y.).

38. Adler J, Lewinson O, \& Bibi E (2004) Role of a conserved membrane-embedded acidic residue in the multidrug transporter MdfA. Biochemistry 43(2):518-525.

39. Kabsch W (2010) Integration, scaling, space-group assignment and postrefinement. Acta Crystallogr D Biol Crystallogr 66(Pt 2):133-144.

40. Vagin AA, Teplyakov, A (1997) MOLREP: an automated program for molecular replacement. J Appl. Cryst. (30):1022-1025.

41. Storoni LC, McCoy AJ, \& Read RJ (2004) Likelihood-enhanced fast rotation functions. Acta Crystallogr. Biol. Crystallogr. D60(Pt 3):432-438.

42. Adams PD, et al. (2010) PHENIX: a comprehensive Python-based system for macromolecular structure solution. Acta Crystallogr D Biol Crystallogr 66(Pt 2):213-221.

43. Emsley P \& Cowtan K (2004) Coot: model-building tools for molecular graphics. Acta Crystallogr. Biol. Crystallogr. D60(Pt 12 Pt 1):2126-2132.

44. Krissinel E \& Henrick K (2004) Secondary-structure matching (SSM), a new tool for fast protein structure alignment in three dimensions. Acta Crystallogr D Biol Crystallogr 60(Pt 12 Pt 1):2256-2268.

\section{Legends to Figures}

Fig. 1. Binding of minocycline $(A)$ and doxorubicin $(B)$ in the deep binding pocket of the $T$ monomer of the AcrB trimer. Binding of the substrate molecules is mainly achieved by aromatic 
1 stacking interactions and polar interactions by the indicated side chains. The mesh represents

2 the $2 \mathrm{~F}_{\mathrm{o}}-\mathrm{F}_{\mathrm{c}}$ electron density map contoured at 1.1 o. (A) AcrB/minocycline complex. The 3 dimethylamino moiety of the drug is sandwiched between F178 and F615. The carboxyamid 4 group interacts with the polar side chain of N274. (B) AcrB/doxorubicin complex. F178 and F615 wedge the $C$ and $D$ rings of doxorubicin. At the other end of the molecule, Q89 interacts with the hydroxyl group of the dihydroxypropanone moiety and also, together with side chain S46, S128 and E130, with the daunosamine sugar moiety.

Fig. 2. Switch-loop conformations of wildtype AcrB, G616N variant and MexB. (A) Cross-eyed stereo representation of the superimposition of the access pocket of the $L$ monomer (blue) and deep binding pocket of the T monomer (yellow). The doxorubicin dimer sandwich is shown in stick and surface representation accommodated in the access pocket on the left. Doxorubicin located in the deep binding pocket in the T monomer (stick and surface representation) is flanked by the PN2 and PC1 subdomains (PC1 subdomain not shown for clarity). The switchloop (including residues F615, F617 and R620 depicted here) adopts different conformations in the L monomer (blue) and T monomer (yellow). The backbone orientation of the switch-loop in the T conformation (yellow) coincides with the doxorubicin dimer sandwich location. Residues Q176 and F615 in the L conformation (blue) are representative for the steric hindrance (caused by the switch-loop and PN2 subdomain, respectively) preventing doxorubicin binding in the deep binding pocket. (B) Superimposition of the wildtype AcrB and G616N variant at the access binding pocket region. Shown on the left is the doxorubicin dimer sandwich (in stick and surface representation) accommodated in the wildtype AcrB $L$ conformation access pocket and the switch-loops of the different conformations in AcrB wildtype ( $L$ conformation, blue, $T$ conformation, yellow) and G616N variant (L conformation, red). The switch-loop of the G616N variant in the $L$ conformation adopts a conformation commensurable with the switch-loop conformation in the AcrB wildtype T conformation. (C) Shown on the left is the doxorubicin dimer sandwich (in stick and surface representation) accommodated in the wildtype AcrB $L$ conformation access pocket and the switch-loops of the G616N variant (red) and MexB (orange) in the $L$ conformation. Both switch-loops of the $G 616 \mathrm{~N}$ variant and MexB are in the $\mathrm{L}$ conformation and adopt a conformation commensurable with the switch-loop conformation in the AcrB wildtype T conformation (see $(B)$ ). MexB and G616N both have substantially lower macrolide efflux activity (30).

Fig. 3. Stepwise AcrB-catalyzed drug transport mediated by cyclic conformations loose (L, blue), tight ( $T$, yellow) and open $(O$, red). Shown are four panels $(A-D)$ indicating the sequential drug 
1 transport steps and conformational cycling of one AcrB monomer within the trimer. The panels

2 show in the upper row a side view of the monomer parallel to the membrane plane, the view is

3 towards the PC1 and PC2 subdomains and the access pocket region. The second row is a view

4 on the porter domain perpendicular to the membrane plane. The third row displays in a

5 trimeric wheel view the state of the monomer from rows one and two (upper left monomer in

6 the wheel, $\mathrm{L}$ in blue, $\mathrm{T}$ in yellow and $\mathrm{O}$ in red) shown in $(A-D)$ in relation to the other monomers

7 in the AcrB trimer. Substrate (doxorubicin) is depicted in green. The putative protonation state

8 of D407 and D408 inside the transmembrane domain is indicated ( $\mathrm{L}$ and T are deprotonated, $\mathrm{O}$

9 is protonated). (A) Initial drug uptake via the access pocket in the L monomer. $(B)$ Transfer of

10 the drug from the low affinity access pocket to the high affinity deep binding pocket during the

$11 \mathrm{~L}$ to $\mathrm{T}$ transition. $(C)$ Directional drug transport during the $\mathrm{T}$ to $\mathrm{O}$ transition. Subdomains PC1

12 and PC2 close the lateral access and prevent backsliding of the drug, which is extruded through

13 tunnel 3. (D) Resetting the monomer by the $O$ to $L$ transition to reinitialize drug uptake via the

14 access pocket. 


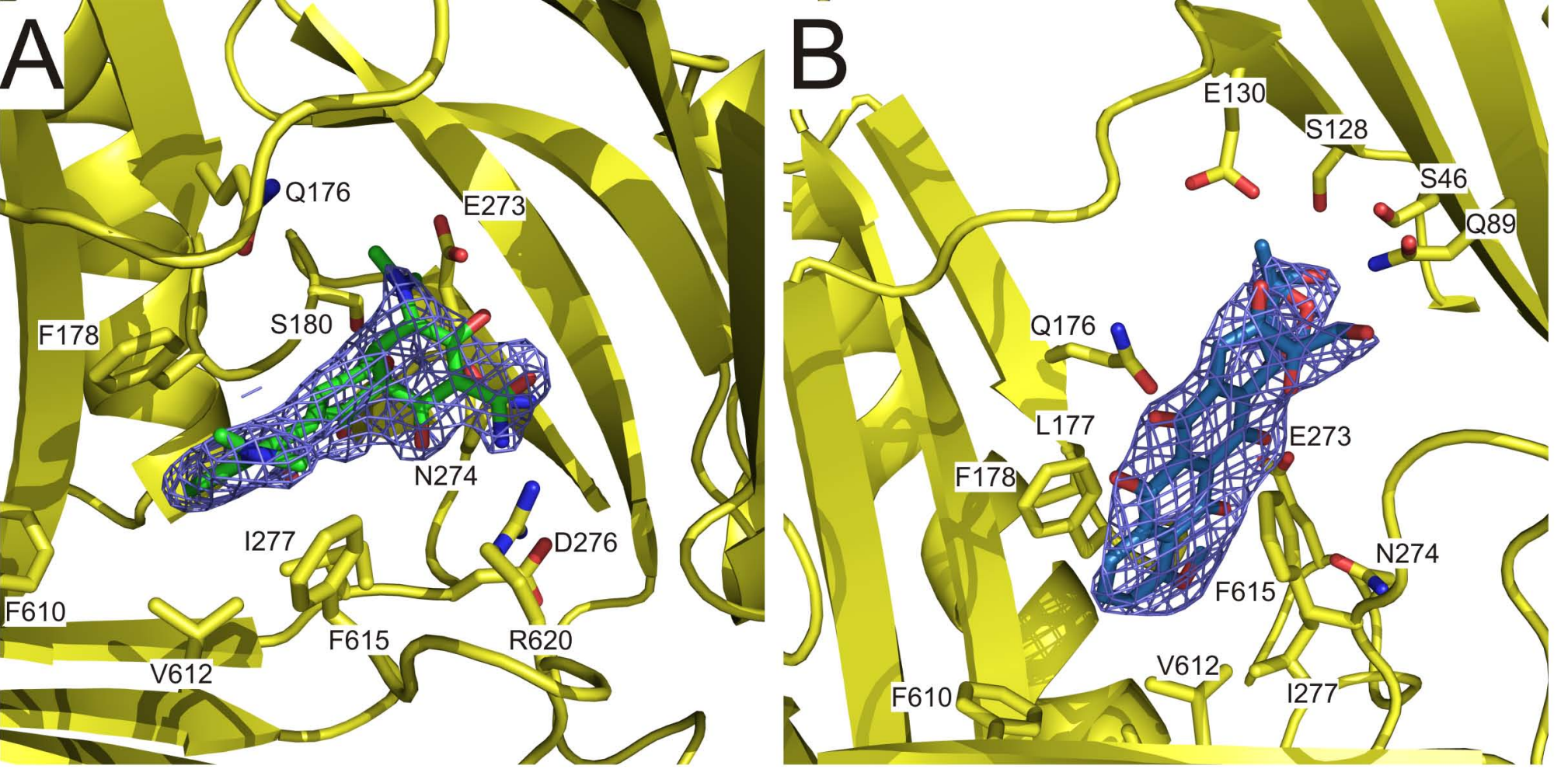

Fig. 1 
side view
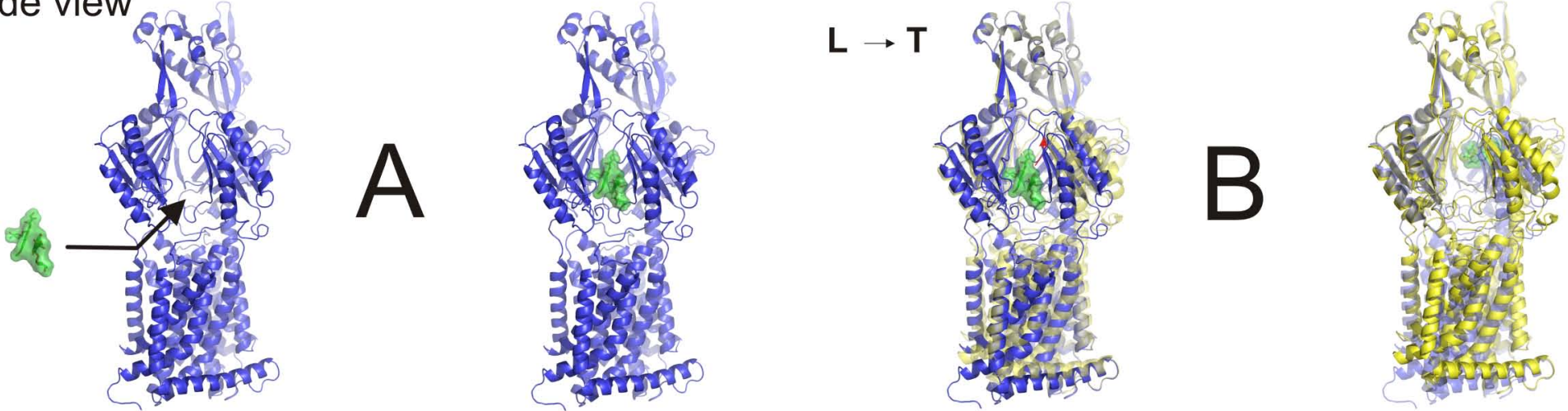

top view
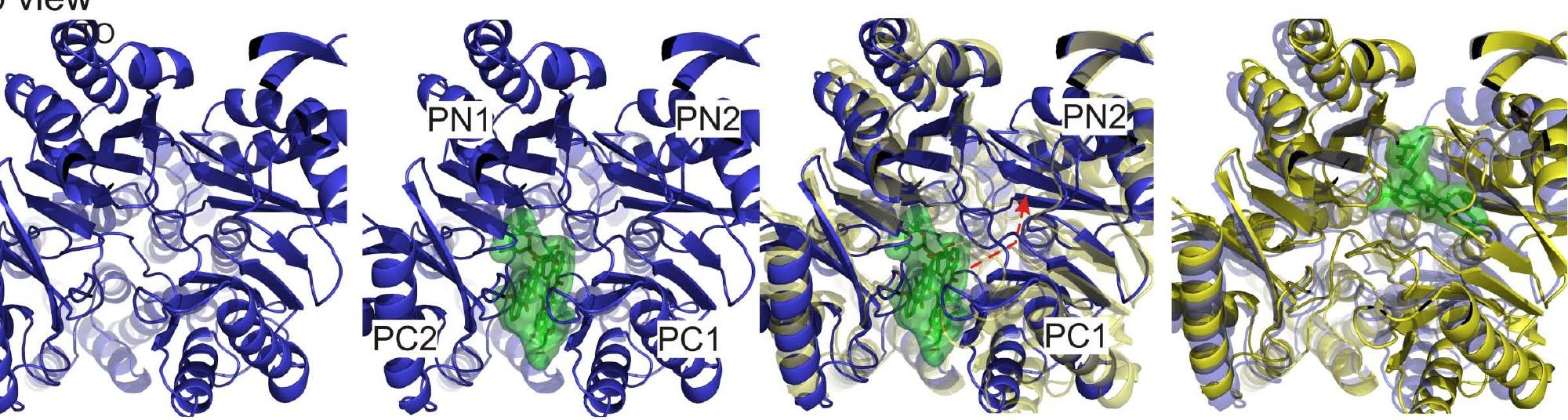

trimeric wheel view
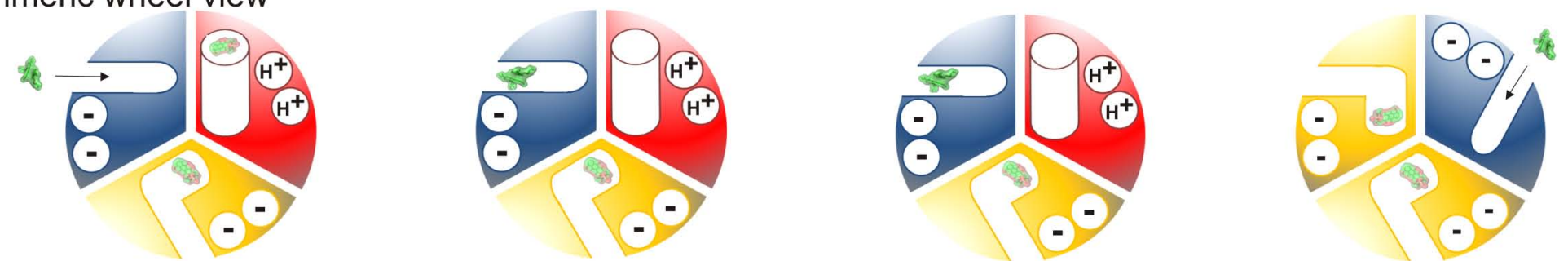

side view

top view
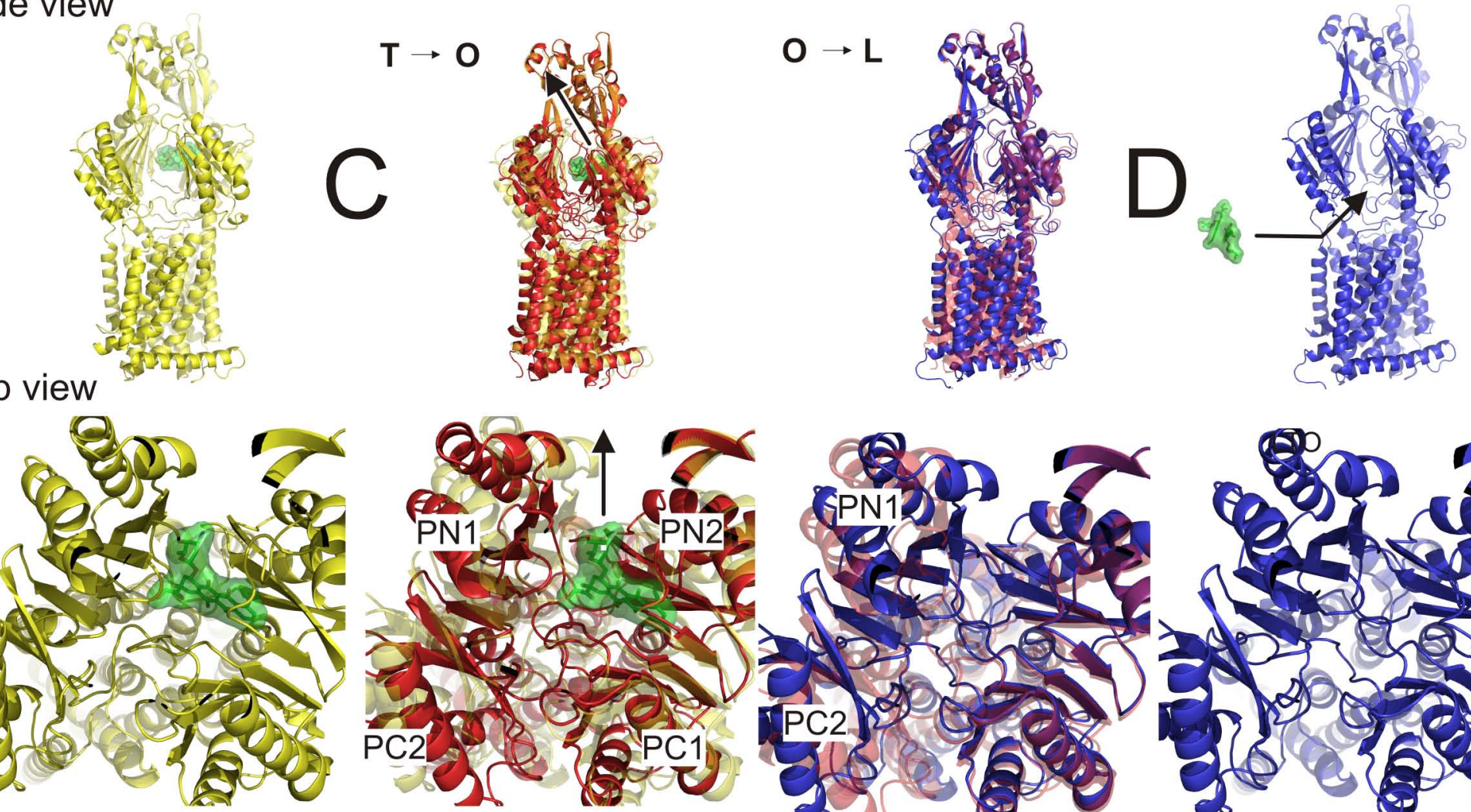

trimeric wheel view
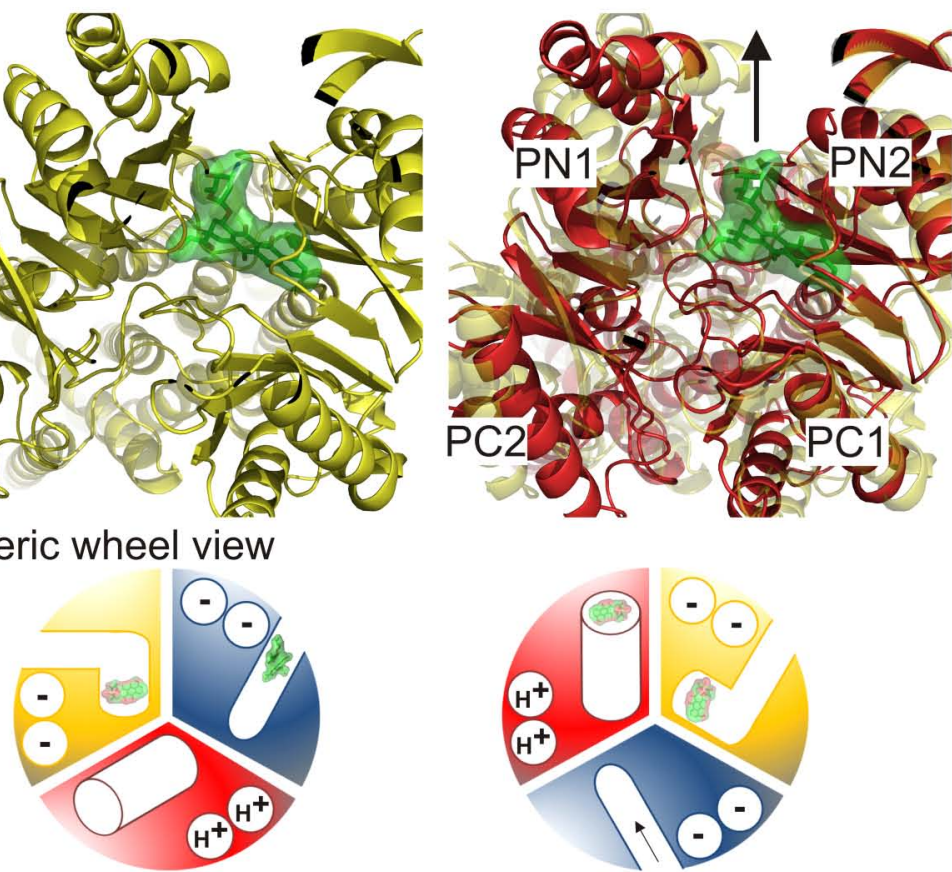

Fig. 3
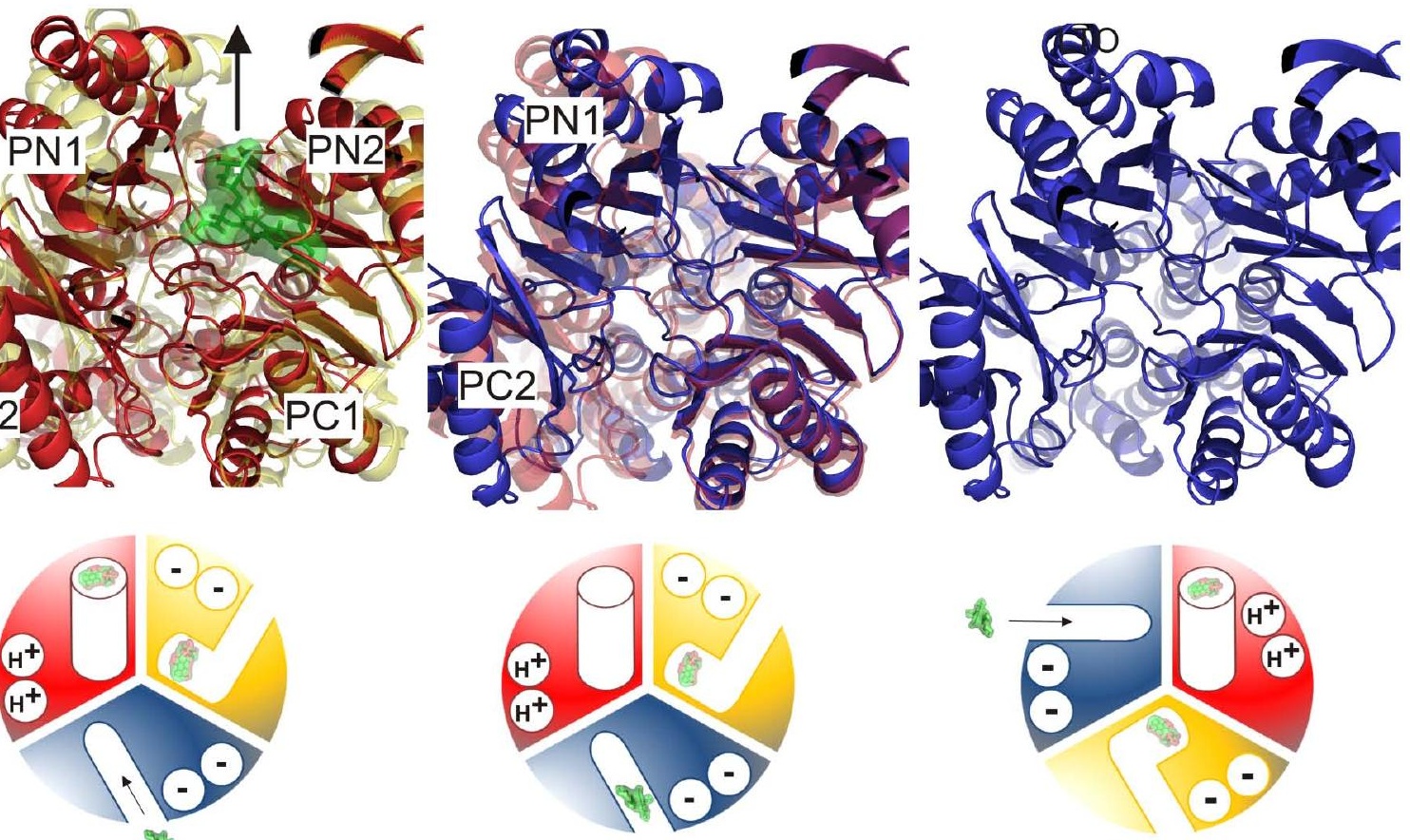
Table S1. Data collection and refinement statistics*

\begin{tabular}{|c|c|c|c|}
\hline & wt_mino & wt_doxo & G616N \\
\hline \multicolumn{4}{|l|}{ Data collection } \\
\hline Space group & $\mathrm{P} 22_{1} 2_{1} 2_{1}$ & $\mathrm{P} 22_{1} 2_{1} 2_{1}$ & $\mathrm{P} 2{ }_{1} 2_{1} 2_{1}$ \\
\hline $\begin{array}{l}\text { Cell dimensions } \\
\text { a, b, c (Å) }\end{array}$ & $145.99,161.74,246.00$ & $145.94,163.29,245.77$ & $145.69,165.45,245.42$ \\
\hline$\alpha, \beta, \gamma\left({ }^{\circ}\right)$ & $90,90,90$ & $90,90,90$ & $90,90,90$ \\
\hline Resolution (Å) & $39.5-1.9(2.01-1.90)$ & $49.1-2.25(2.39-2.25)$ & $50-2.89(3.07-2.89)$ \\
\hline$R_{\text {meas }}$ & $12.5(260.2)$ & $10.2(109.4)$ & $22.9(159.5)$ \\
\hline $1 / \sigma_{1}$ & $12.4(0.7)$ & $14.9(1.8)$ & $10.2(1.4)$ \\
\hline Completeness (\%) & $99.0(94.2)$ & $99.5(97.3)$ & $99.7(98.6)$ \\
\hline Multiplicity & $6.9(4.4)$ & $7.0(5.2)$ & $7.5(7.4)$ \\
\hline \multicolumn{4}{|l|}{ Refinement } \\
\hline Program & Phenix v. dev. 985 & Phenix v. dev. 985 & Phenix v. dev. 985 \\
\hline Resolution (Å) & $39.5-1.90(1.92-1.90)$ & $49.1-2.25(2.28-2.25)$ & $49.3-2.9(2.93-2.90)$ \\
\hline No. reflections & 450986 & 274555 & 131572 \\
\hline $\mathrm{R}_{\text {work }} / \mathrm{R}_{\text {free }}$ & 20.0/23.1 (43.9/45.9) & $18.5 / 22.7(31.2 / 33.9)$ & $21.0 / 27.1(33.6 / 38.1)$ \\
\hline \multicolumn{4}{|l|}{ No. atoms } \\
\hline Protein & 25969 & 25979 & 25987 \\
\hline Ligands and ions & $\begin{array}{l}1258 \\
8 \text { LMT (dodecyl- } \beta \text {-D-maltoside) } \\
1 \text { LMU (dodecyl- } \alpha \text {-D-maltoside) } \\
26 \text { C14, D12, UND, D10, DD9, OCT, } \\
\text { HEX (alkane chains) } \\
1 \text { MIY (minocycline) } \\
15 \text { GOL (glycerol) } \\
1 \text { SO4 (sulfate ion) }\end{array}$ & $\begin{array}{l}788 \\
7 \text { LMT (dodecyl- } \beta \text {-D-maltoside) } \\
1 \text { LMU (dodecyl- } \alpha \text {-D-maltoside) } \\
3 \text { DM2 (doxorubicin) } \\
10 \text { D12, D10, HEX (alkane chains) } \\
3 \text { GOL (glycerol) }\end{array}$ & $\begin{array}{l}245 \\
7 \text { LMT (dodecyl- } \beta-D-m a l t o s i d e)\end{array}$ \\
\hline Water & 1783 & 1700 & - \\
\hline \multicolumn{4}{|l|}{ B-factors $\left(\AA^{2}\right)$} \\
\hline Average & 45.3 & 51.3 & 61.8 \\
\hline Protein & 44.4 & 50.4 & 61.3 \\
\hline Water & 45.6 & 53.0 & - \\
\hline \multicolumn{4}{|l|}{ R.M.S. deviations } \\
\hline Bond lengths $(\AA)$ & 0.007 & 0.007 & 0.009 \\
\hline Bond angles $\left({ }^{\circ}\right)$ & 1.09 & 1.05 & 1.26 \\
\hline
\end{tabular}


residues

distance

$(\AA)^{a}$

Doxorubicin (DM2)

S46 (CB) - DM2 (O4')

Q89 (NE2) - DM2 (O5')

S128 (OG) - DM2 (O4')

E130 (OE1) - DM2 (C4')

Q176 (OE1) - DM2 (C8)

F178 (CD1) - DM2 (C20)

G179 (N) - DM2 (C21)

1277 (CB) - DM2 (O4)

F610 (CZ) - DM2 (C2)

V612 (CG2) - DM2 (C2)

F615 (CE1) - DM2 (O13)
3.2

3.3

5.0

3.2

3.5

3.2

3.0

4.5

4.7

3.8

3.7 residues

distance $(\AA)^{\mathrm{a}}$
Minocycline (MIY)

\footnotetext{
${ }^{a}$ : shortest interatomic distance
} 
Fig. S1. $(A-D)$ Structural features of trimeric AcrB (pdb entry: 1IWG). The monomers are colored in different shades of blue. $(A)$ The AcrB trimer (side view, cartoon representation) consists of a transmembrane domain, the periplasmic porter domain and periplasmic TolC docking domain (as indicated on the left). The central part of the porter domain separates the funnel from the central cavity. $(B)$ Side view of the AcrB trimer in surface representation. The central cavity (see $A)$ ) is accessible to solvent via the three vestibules formed in each case at the interface of two monomers. Other potential substrate binding sites, i.e. the PC1/PC2 cleft at the periplasmic porter domain and the TM8/TM9 groove in the transmembrane domain are indicated. $(C)$ Topological view of the transmembrane domain of trimeric AcrB viewed from the periplasm. Each monomer consist of 12 TM helices with central TM helix 4 and 10 harbouring three of the four essential charged residues (D407, D408 and K940, not shown is R971 on TM11). The three monomers define the central cavity (see also $A)$ ). (D) Periplasmic top-view of the AcrB porter domain with subdomains PN1, PN2, PC1 and PC2. The three central $\alpha$-helices (one from each PN1 subdomain) define a central pore structure (arrow) separating the funnel from the central cavity (as shown in A)). At the periphery, each monomer harbours a cleft formed at the interface of the PC1 and PC2 subdomains (see also $B)$ ). ( $E-G$ ) Visualization of tunnels (in green) in the porter domain of the different monomers (pdb entry 2J8S). (E) In the L monomer (blue), tunnel 2 leads from the access site situated in the PC1/PC2 cleft (see B and D) about $15 \AA$ above the membrane plane towards the centre of the periplasmic porter domain. $(F)$ An additional tunnel (tunnel 1 ) is apparent in the T conformer (yellow). This tunnel starts at the height of the membrane plane exactly at the periplasmic end of the TM8/TM9 groove (see $B$ ) and joins tunnel 2 near the deep binding pocket in the T conformer. $(G)$ Due to substantial reorientation of the porter subdomains and the coil-to-helix transition at the $\mathrm{N}$-terminal end of TM8, both lateral tunnels collapse in the $\mathrm{O}$ conformer (red). Another tunnel (tunnel 3 ) is created instead and leads from the now closed binding pocket to the funnel in the centre of trimer (see $A$ ).

Fig. S2. Superimposition of bound minocycline and doxorubicin in the deep binding pocket of the AcrB T monomer. (A) Superimposition of doxorubicin binding in 2DR6 at $3.3 \AA$ resolution (1) and the current structure at $2.2 \AA$ resolution (in yellow and doxorubicin in cyan, doxorubicin and amino acid side chains of the 2DR6 model that differ substantially are indicated in grey). (B) Superimposition of the AcrB co-crystal 
structures with minocycline (green) and doxorubicin (cyan) in the deep binding pocket of the AcrB $T$ monomer (yellow). The side chains shown here are from the AcrB/minocycline co-crystal structure.

Fig. S3. Binding of doxorubicin in the deep binding pocket and of dodecyl- $\alpha-D-$ maltoside in the lateral binding pocket of the T monomer of the AcrB trimer. $(A)$ Side view of the AcrB T monomer parallel to the membrane plane. The view is towards the PC1 and PC2 subdomains. (B) Close-up cross-eyed stereo representation of doxorubicin and dodecyl- $\alpha$-D-maltoside binding. Side-chain interaction (all residues within $3.5 \AA$ distance are shown) with dodecyl- $\alpha$-D-maltoside is mainly by aromatic stacking interactions (F666) and polar interactions (D566). The blue mesh represents the $2 F_{0}-F_{c}$ electron density map at $1.0 \sigma$.

Fig. S4. Binding of doxorubicin in the lateral access pocket of the $L$ monomer. $(A)$ Side view of the $\mathrm{L}$ monomer in cartoon representation with two doxorubicin molecules bound to the access pocket. Inset: Close up of the access pocket with $F_{0}-F_{c}$ densities observed (red mesh) at $3 \sigma .(B)$ Close up of the access pocket with two doxorubicin molecules shown in stick representation (green, oxygen atoms in red). Amino acid side chains involved in Bodipy-FL-maleimide labeling after Cys-substitution (2) are shown as red sticks. $(C)$ region view as in $(B)$ in cross-eyed stereo representation of the access pocket in the $\mathrm{L}$ monomer with two doxorubicin molecules shown in stick representation (green, oxygen atoms in red) and $2 F_{o}-F_{c}$ densities (blue mesh) after refinement at $1 \sigma$. Indicated in figures $A-C$ are side chain residues (stick representation, blue, oxygen atoms in red) within $3.5 \AA$ distance of the doxorubicin molecules.

Fig. S5. Localization of the access and deep binding pockets with bound doxorubicin in the asymmetric AcrB trimer. The monomers are colored in blue ( $L$ monomer), yellow $(T)$ or red (O). Tunnels (see Figure S1) are visualized in green. $(A)$ Co-crystal structure of the asymmetric AcrB trimer (side view, cartoon representation) with two doxorubicin molecules (yellow sphere representation) bound to the access pocket in the $L$ monomer and in the same trimer one doxorubicin (blue spheres) bound at the deep binding pocket in the T monomer. $(B)$ Periplasmic top-view of the asymmetric AcrB trimer (representation of $(A)$ drawn 90 degrees 
towards the viewer) with bound doxorubin molecules to the access and deep binding pockets. The switch loops in the $L$ and $T$ monomer are indicated in red.

Fig. S6. $(A, B)$ Binding of dodecyl- $\beta-D-$ maltoside in the TM8/TM9 groove of the $L$ monomer and proximity to the stacked doxorubicin molecules in the access pocket. $(A)$ Side view of the $L$ monomer in cartoon representation (blue). Orientation is identical as in Figure S4A. Location of the bound dodecyl- $\beta$-D-maltoside in the TM8/TM9 region is shown in relation to the bound doxorubicin sandwich in the access pocket of the $L$ monomer. Dodecyl- $\beta$-D-maltoside and doxorubicin are presented as sticks (green, oxygen atoms in red). (B) Close up of the TM8/TM9 region. Shown are all side chain residues (stick representation in blue, oxygen atoms in red) within $3.5 \AA$ distance of dodecyl- $\beta$-D-maltoside (green stick representation, oxygen atoms in red). Shown in grey mesh is the $2 \mathrm{~F}_{\mathrm{o}}-\mathrm{F}_{\mathrm{c}}$ density after refinement at $1 \sigma$ (2.26 $\AA$ resolution). $(C, D)$ Omit maps for the switch-loop region (amino acid residues 614-622) in the $L$ monomer of $(C)$ AcrB/doxorubicin co-crystal structure (blue stick representation) and $(D) \mathrm{G} 616 \mathrm{~N}$ crystal structure (red stick representation). The $F_{0}-F_{c}$ densities are indicated in green mesh at $3 \sigma$. The presented switch loop regions were built on basis of the $F_{0}-F_{c}$ and $2 F_{0}-F_{c}$ densities.

Fig. S7. Binding of ethidium, taurocholate or doxorubicin to the PC1/PC2 cleft. Superimposition of the AcrB porter domain (subdomains PN1, PN2, PC1 and PC2) of monomers derived from the symmetric AcrB structures 1T9X (3)(red, $3.1 \AA$ ), 2W1B (4) (green, $3.85 \AA$ ) (co-crystal structures with ethidium and taurocholate respectively) and the $L$ monomer of the asymmetric doxorubicin/AcrB co-crystal structure (blue, $2.26 \AA$ ). $(A)$ Localization of two doxorubicin molecules (blue sphere representation) in the access pocket of the $\mathrm{L}$ monomer of asymmetric AcrB (blue cartoon). (B) Localization of ethidium (red spheres) or taurocholate (green spheres) at each of the monomers of symmetric AcrB (1T9X, red and 2W1B, green) at a more peripheral site in the PC1/PC2 cleft. (C) Superimposition of the AcrB porter domain of co-crystal structures 1T9X (with ethidium, red), 2W1B (with taurocholate, green) and the AcrB/doxorubicin co-crystal structure (blue) and localization of ethidium (red spheres), taurocholate (green spheres) and doxorubicin ( 2 molecules, blue spheres) at the PC1/PC2 cleft. The switch-loop positions are indicated by the arrows. 
Fig. S8. (A) Antibiotic resistance activity of AcrB wildtype, AcrB D407N_D408N, or AcrB G616N in an E. coli BW25113 $\triangle a c r B$ background. Growth in the presence of chloramphenicol $\left(1 \mu \mathrm{g} \mathrm{ml} l^{-1}\right)$, Doxorubicin $\left(10 \mu \mathrm{g} \mathrm{ml}^{-1}\right)$, Erythromycin $\left(10 \mu \mathrm{g} \mathrm{ml}{ }^{-1}\right)$, Novobiocin $\left(5 \mu \mathrm{g} \mathrm{ml} l^{-1}\right)$, Ethidium $\left(50 \mu \mathrm{g} \mathrm{ml}^{-1}\right)$ and in absence of drug (control) was analyzed on solid LB-agar medium containing $50 \mu \mathrm{g} \mathrm{ml}^{-1}$ kanamycin as selective drug for the $\mathrm{pET} 24$ plasmid carrying the AcrB (wildtype or variant) gene. Cells ( $4 \mu$ of an overnight culture adjusted to an $\mathrm{OD}_{600}$ of 1 ) were diluted as indicated and spotted onto the LB agar plate containing the indicated antibiotics. AcrB D407N_D408N contains substitutions in the putative proton translocation site of AcrB and was previously shown to be devoid of activity for all tested substrates (5).

(B) Western blot analysis of cell extracts obtained from E. coli BW25113 $\Delta a c r B$ harbouring plasmids encoding wildtype AcrB, G616N variant, D407N_408N variant or no AcrB (control vector pET24). Cell extracts were obtained from cells growing on solid LB-agar medium as described above, diluted to an $\mathrm{OD}_{600}$ of 1.0 in LB-medium and treated as described previously using anti-AcrB rabbit antibodies for immunodetection (5).

1. Murakami S, Nakashima R, Yamashita E, Matsumoto T, \& Yamaguchi A (2006) Crystal structures of a multidrug transporter reveal a functionally rotating mechanism. Nature 443(7108):173-179.

2. Husain F \& Nikaido H (2010) Substrate path in the AcrB multidrug efflux pump of Escherichia coli. Mol Microbiol 78(2):320-330.

3. Yu EW, Aires JR, McDermott G, \& Nikaido H (2005) A periplasmic drug-binding site of the AcrB multidrug efflux pump: a crystallographic and site-directed mutagenesis study. J. Bacteriol. 187(19):6804-6815.

4. Drew D, et al. (2008) The structure of the efflux pump AcrB in complex with bile acid. Mol Membr Biol 25(8):677-682.

5. Seeger MA, et al. (2008) Engineered disulfide bonds support the functional rotation mechanism of multidrug efflux pump AcrB. Nat Struct Mol Biol 15(2):199-205. 
B

TolC docking domain

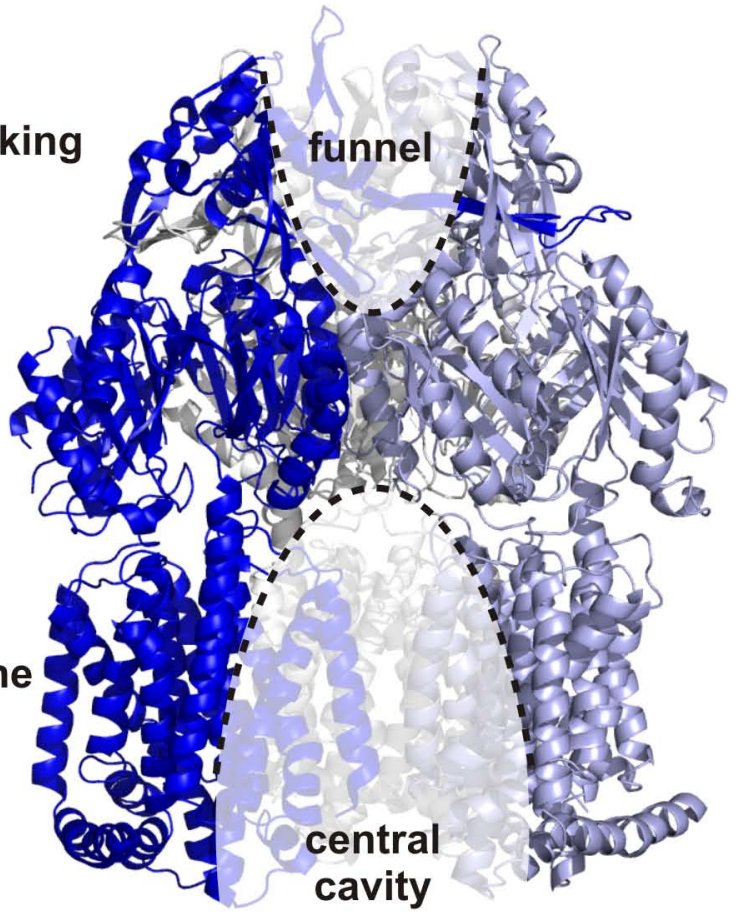

porter domain tra
m
do
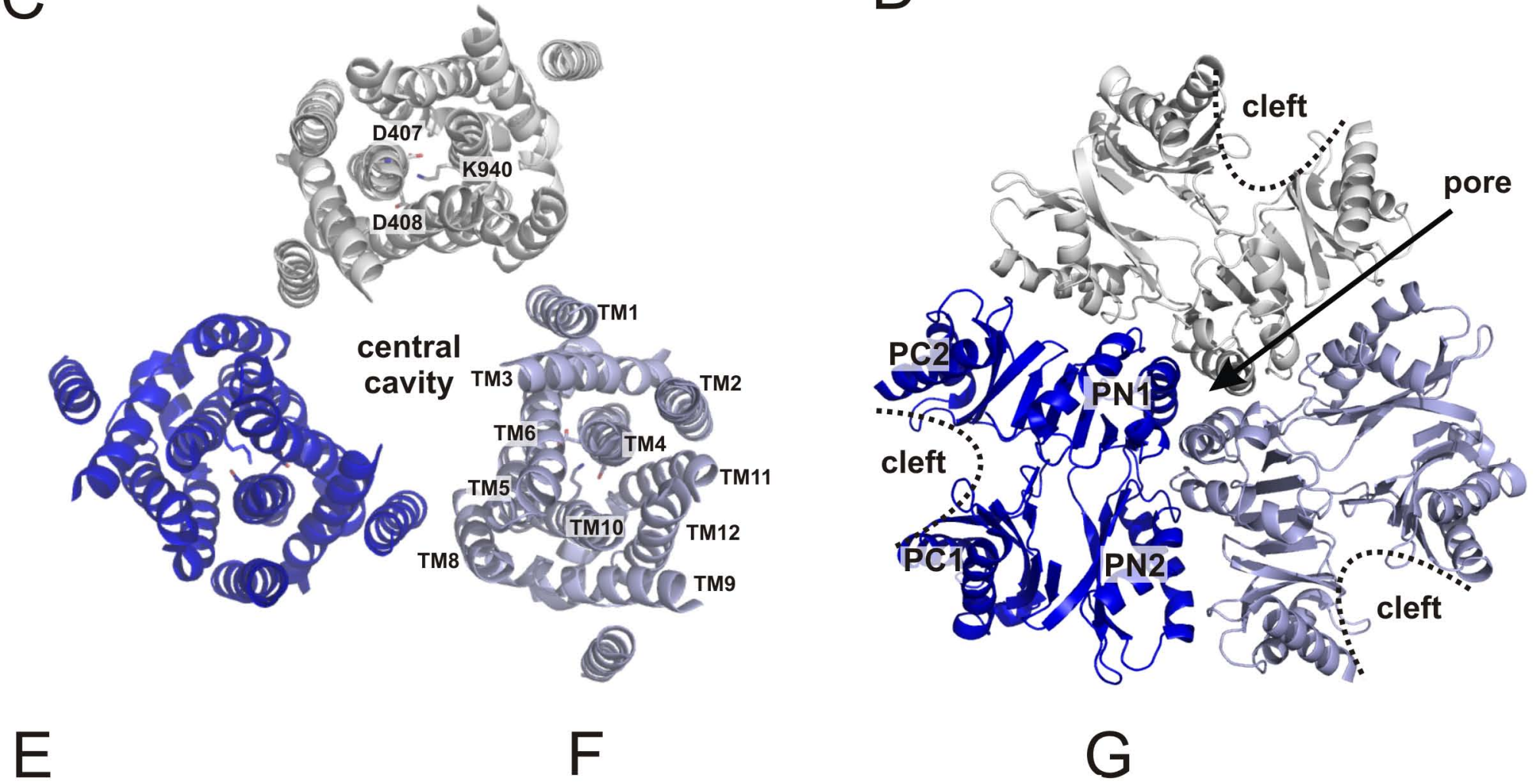

G
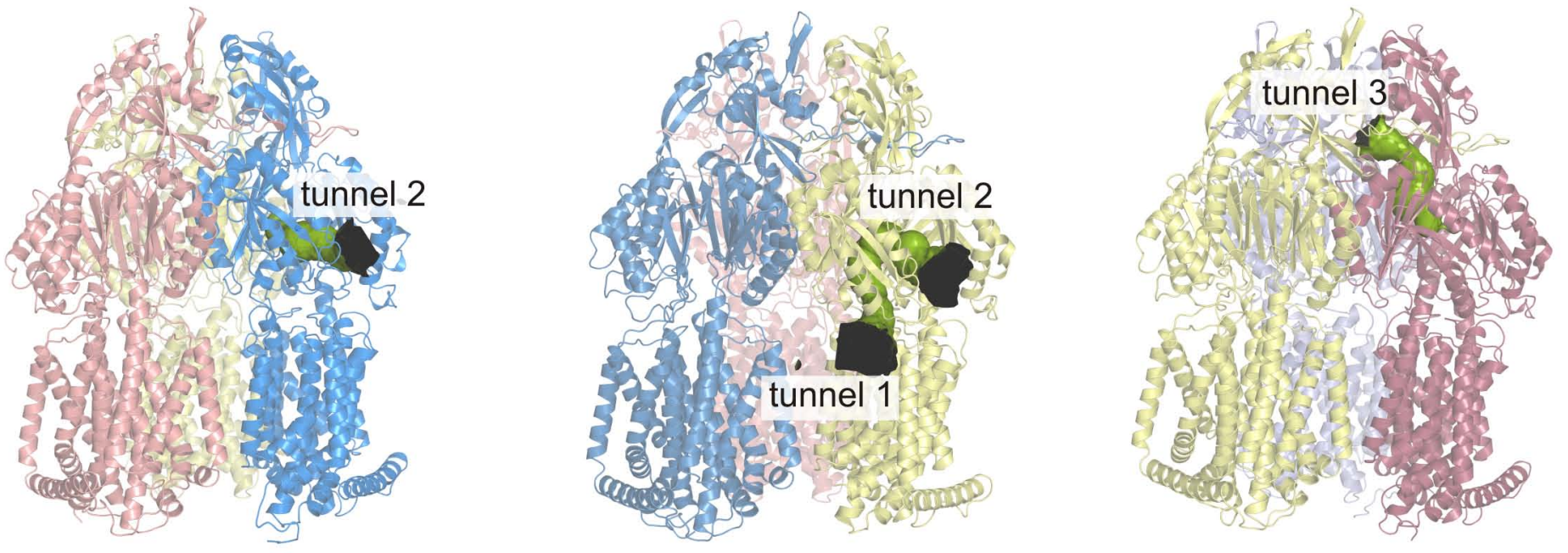

Fig. S1 
$A$
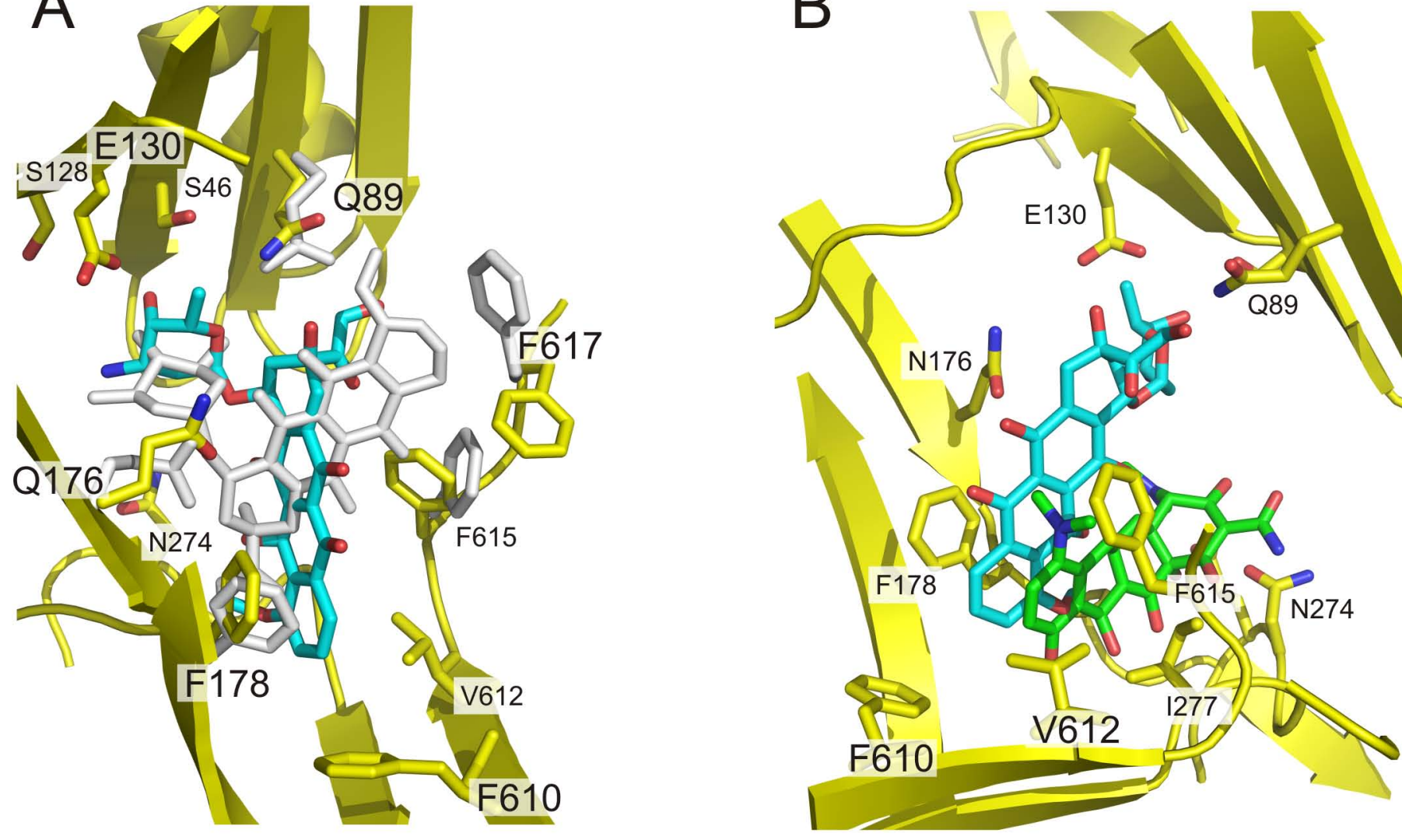
A

B

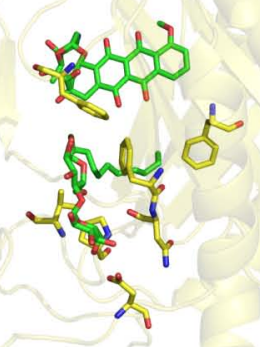

Fig. S3

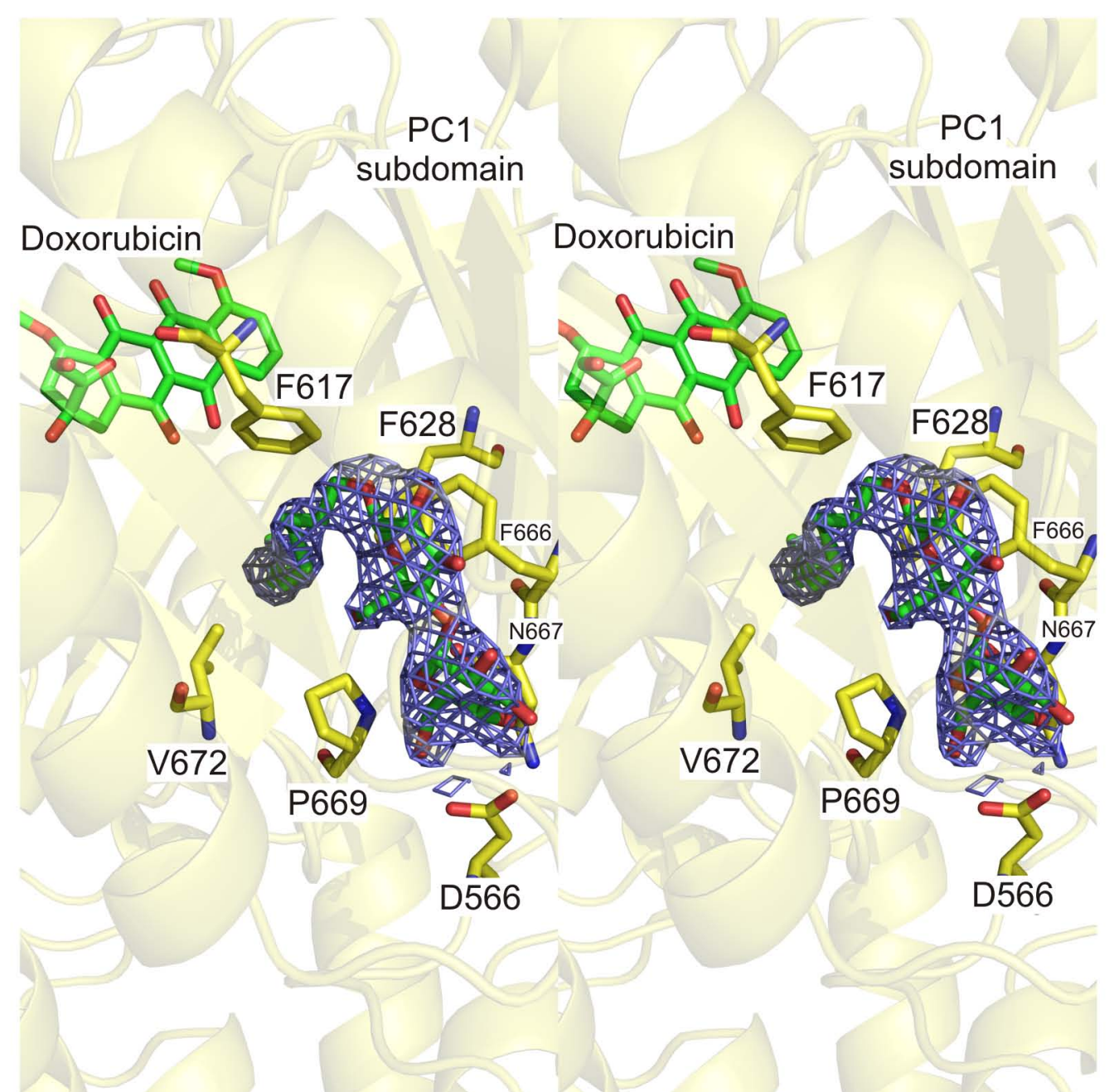




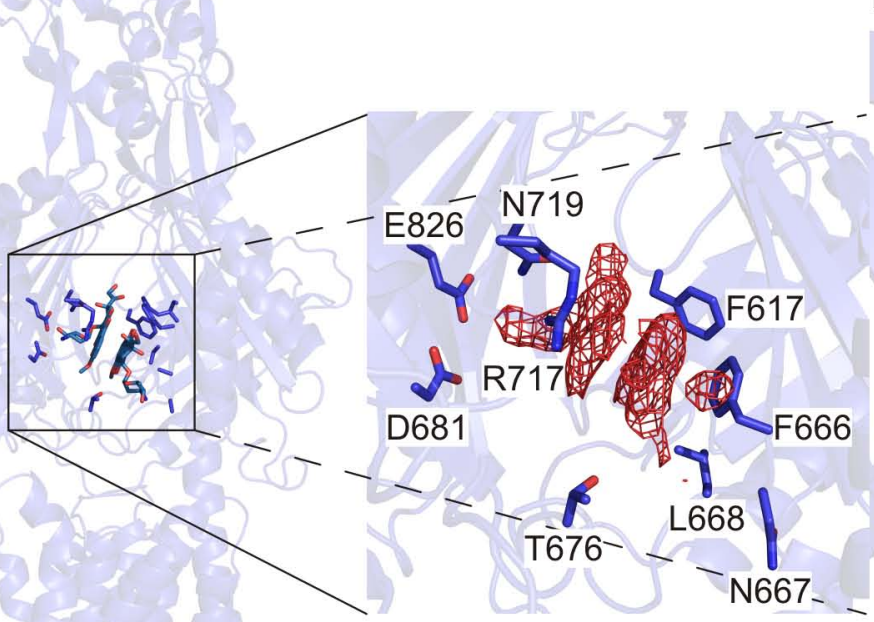

E826

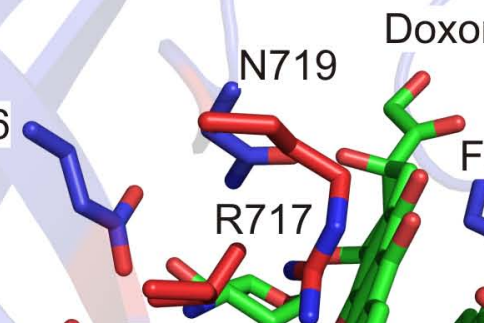

rubicin
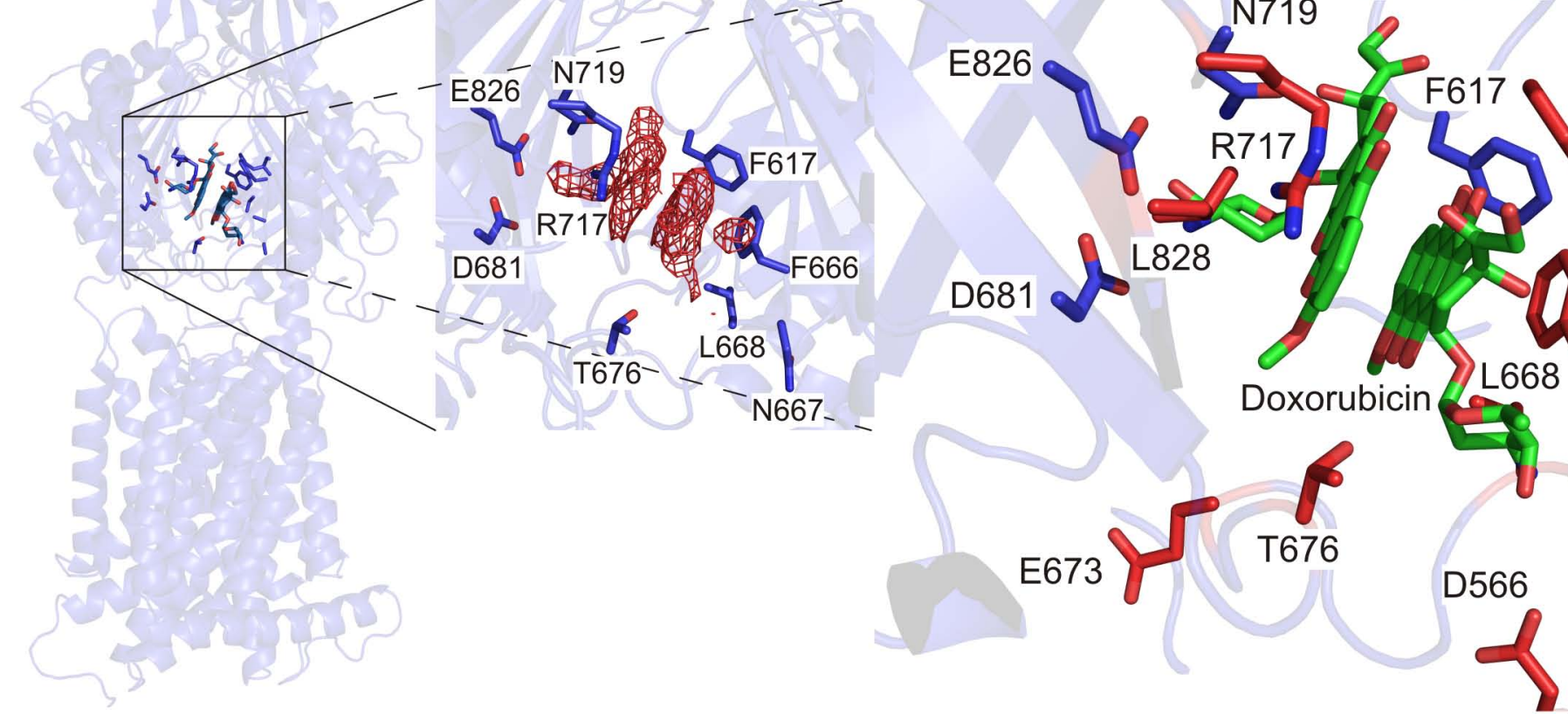

C
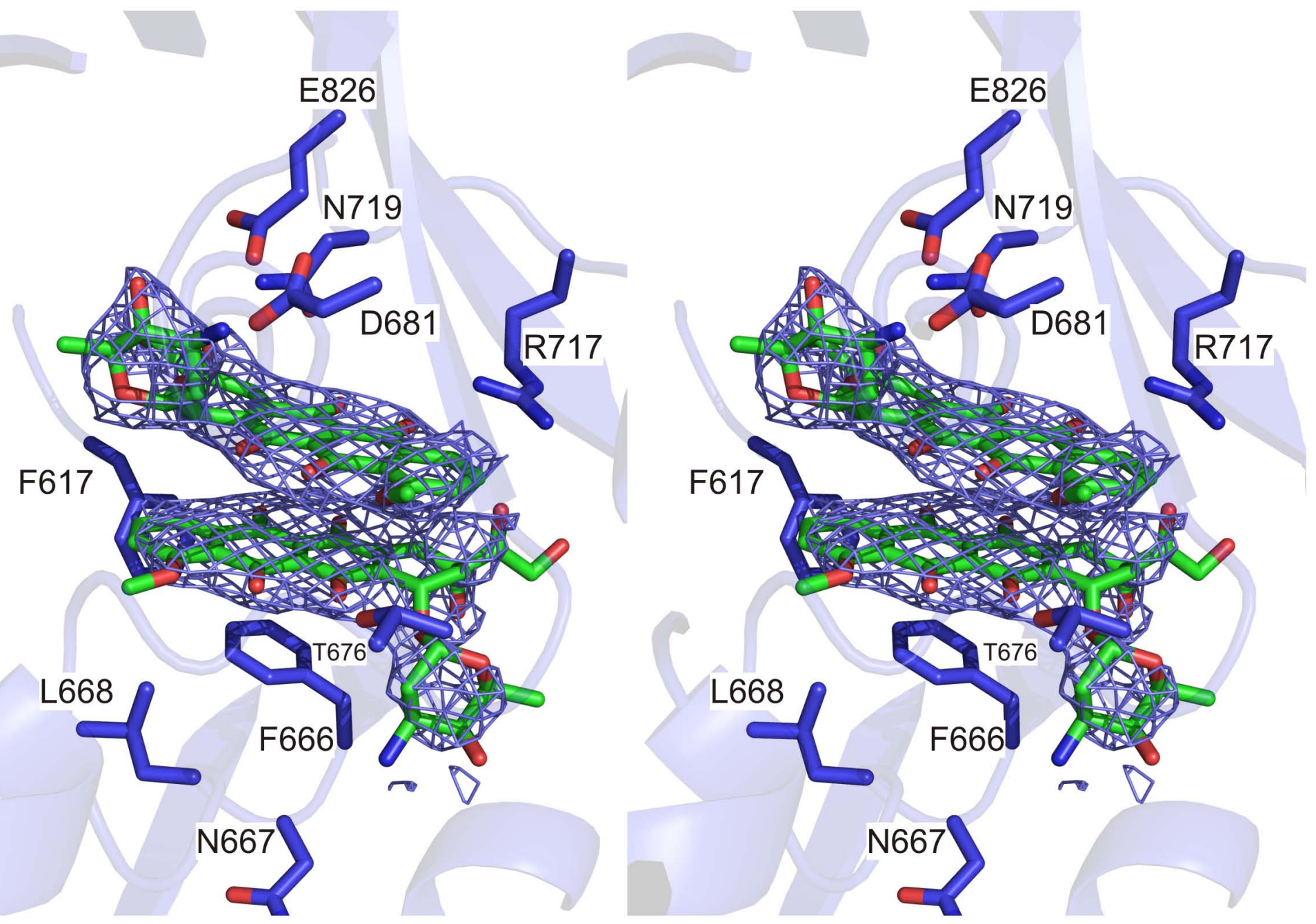

Fig. S4 


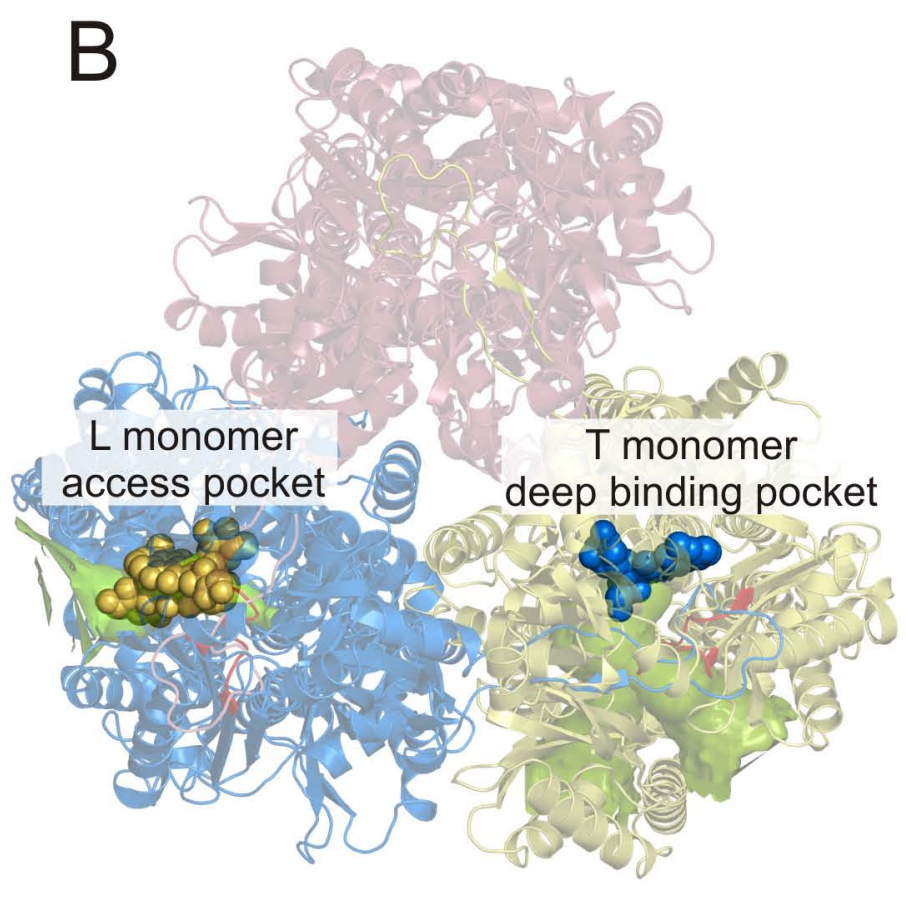

\section{Fig. S5}



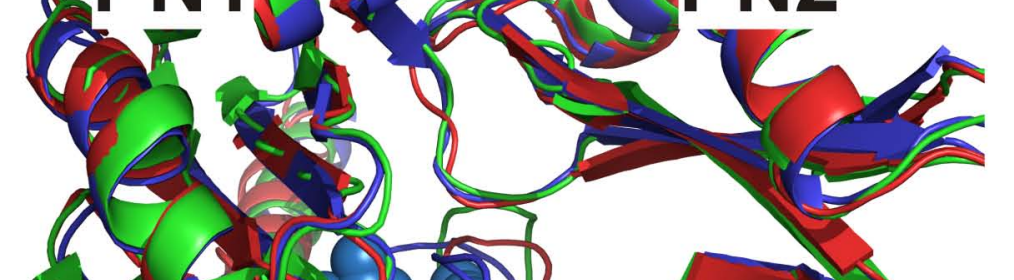

(6).

$162=$

2PC2 10.1010 Sc Doxorution?

BAP1 SLPA1

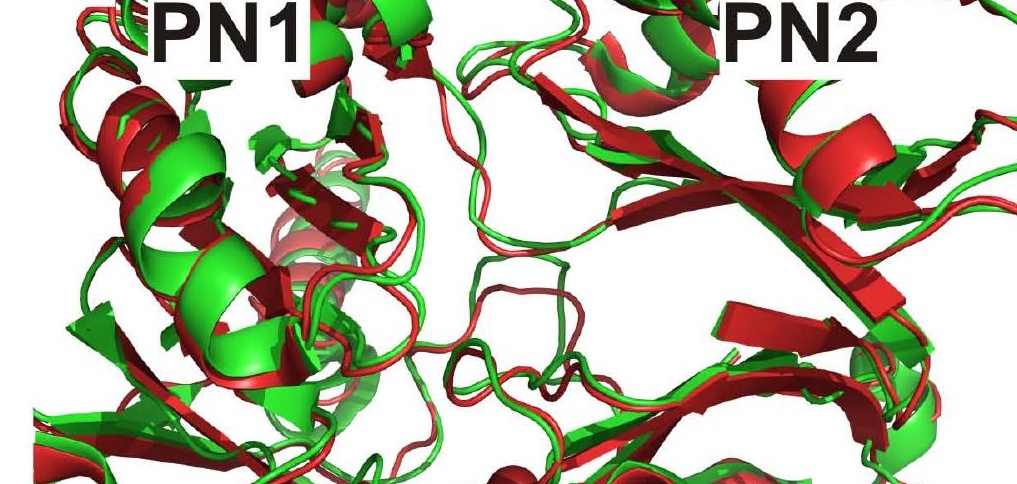

$e^{2}$

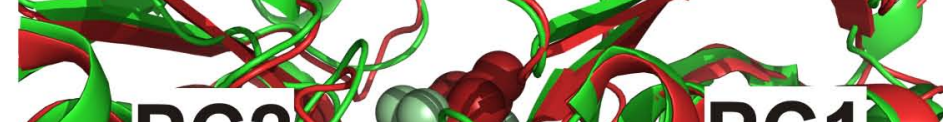

PC2 95 PC10

104 Ethidium Taurocholate

C

C

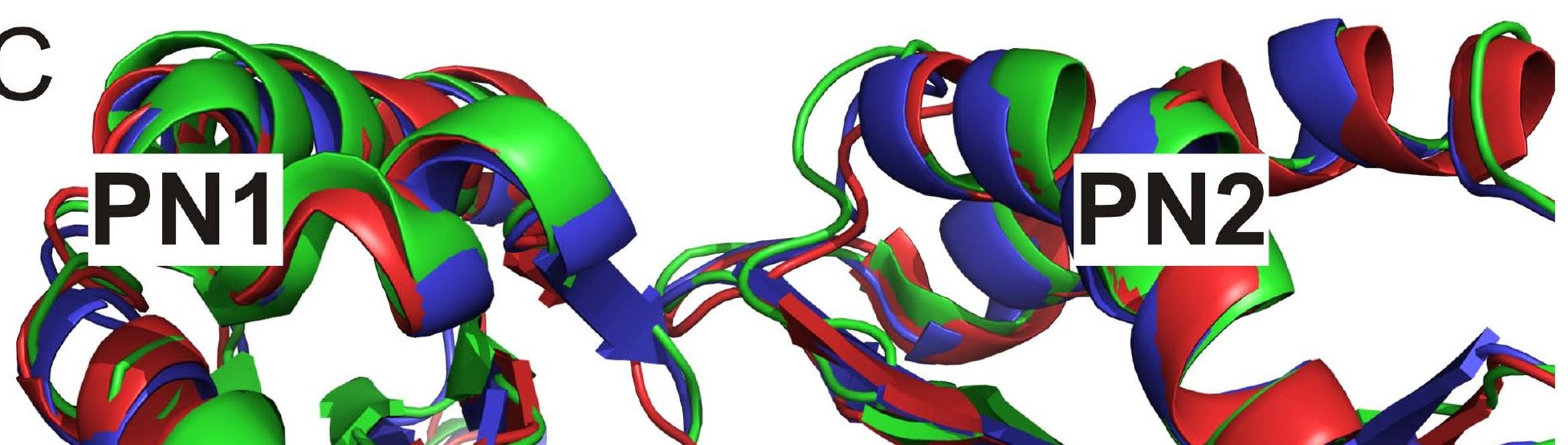

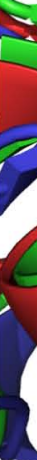

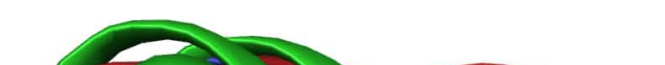

Fig. S7

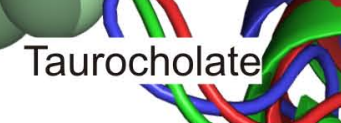




$$
\begin{array}{llllllllllllllllll}
\mathrm{x}=1 & 2 & 3 & 4 & 5 & 6 & 1 & 2 & 3 & 4 & 5 & 6 & 1 & 2 & 3 & 4 & 5 & 6
\end{array}
$$
AcrB D407N_D408N AcrB wildtype AcrB G616N

Chloramphenicol Doxorubicin Erythromycin Dilution $\left(10^{-x}\right)$

$$
\begin{array}{llllllllllllllllll}
x=1 & 2 & 3 & 4 & 5 & 6 & 1 & 2 & 3 & 4 & 5 & 6 & 1 & 2 & 3 & 4 & 5 & 6
\end{array}
$$
AcrB D407N_D408N AcrB wildtype AcrB G616N

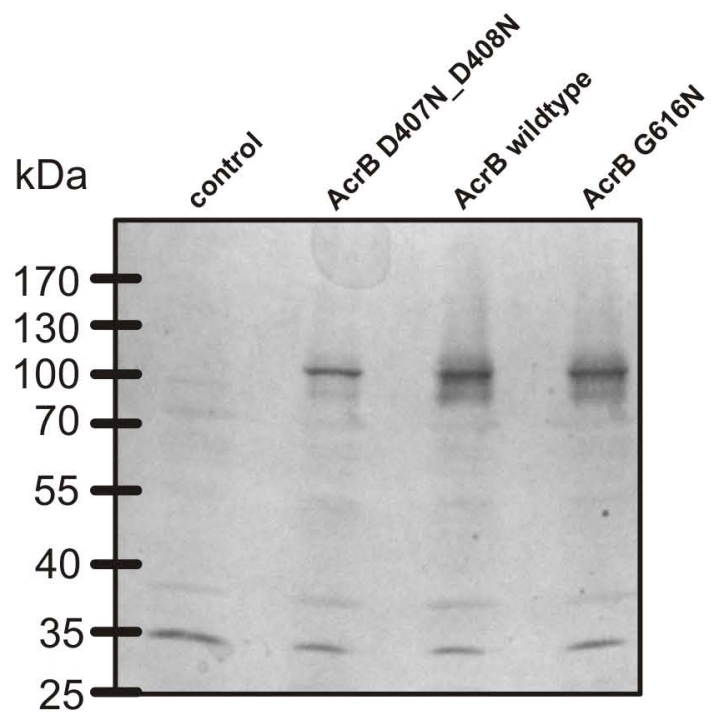

